\title{
Renormalization Group and the Ultraviolet Problem in the Luttinger Model
}

\author{
G. Gentile and B. Scoppola \\ Dipartimento di Fisica, Università di Roma, P.le A. Moro 5, I-00185 Roma, Italy
}

Received September 25, 1992

\begin{abstract}
The Luttinger model describes a non-local interacting relativistic theory for spinless and massless fermions. Albeit the exact solution is already known, the perturbative approach to the model via the renormalization group is useful on account of the connection to the study of more realistic models' behaviour near the Fermi surface. In this work we show that the effective potential describing the interaction on the physical scale $p_{0}^{-1}$ is analytical in the coupling constants, and has an exponential decay on that scale. Besides the physical motivation of this approach, the problem is also technically interesting, since it is an example of a trivially superrenormalizable theory, as far as the ultraviolet region is concerned; nevertheless the proof is quite delicate, as the convergence of the perturbative series does not follow from the superficial bounds (which would give logarithmic and linear divergences), but is due to accidental compensations furnished by the particular symmetry properties of the model.
\end{abstract}

$$
\begin{aligned}
& \text { Tyger, Tyger, burning bright } \\
& \text { In the forests of the night, } \\
& \text { What immortal hand or eye } \\
& \text { Could frame thy fearful symmetry? }
\end{aligned}
$$

William Blake

\section{Introduction}

Recently a considerable effort has been spent in the study of one dimensional models of interacting fermions in the framework of the perturbative theory. The physical reason of this interest is due to the modifications that the interaction brings to the Fermi surface (anomalous Fermi surface) and to the possible relation with the models in higher dimensions. From the mathematical point of view such models, despite their one dimensionality, have a very rich structure because some basic features related to the anomalous behaviour, which can be straightforward to understand in a more qualitative approach, become much more complicated to prove in a rigorous way. 
If one considers the exactly soluble Luttinger model [L, ML], in the perturbative theory, it is formally possible to show directly that the $\beta$ function vanishes identically (see $[\mathrm{DM}]$ ), by using the symmetries of the model. In the rigorous approach proposed by $[B G]$, however, the proof of the same property becomes quite indirect, and uses also the results of the exact solution of the model (see [BGM]). The vanishing of the $\beta$ function of the Luttinger model is, on the other hand, a basic tool in the proof of the analyticity of the Schwinger functions of a more realistic, and not exactly soluble, model of interacting Fermions (see [BGM, BGPS]).

In [BGM, BGPS] the proofs of the analyticity of the Schwinger functions and of the vanishing of the $\beta$ function for the realistic model and for the Luttinger model are given assuming that no divergence arises in the perturbative approach to the ultraviolet region of the Luttinger model. This is needed in order to insure that the problem attached with the perturbative approach is truly the exactly soluble one. Physically this is reasonable, since the coupling constants depend only on the features of the problem near the Fermi surface. On the other hand this is not obvious because only the infrared region of the Luttinger model admits an immediate physical interpretation, while the exact solution contains the details on every scale.

Moreover the solution of the ultraviolet problem, which is given in this work, is technically interesting and shows how the detailed knowledge of the perturbative expansion given by the tree graph technique (see $[\mathrm{G} 1, \mathrm{GN}]$ ) allows us to cancel the superficial divergences of the theory.

The main problem that we met in this work is the following. In the Luttinger model the interaction between particles is non-local, but has finite range $p_{0}^{-1}$ [see (1.4) below]. This allows us to distinguish in a natural way the ultraviolet and the infrared region of the problem: actually the interaction potential can be regarded as local for the space scales bigger than $p_{0}^{-1}$ (infrared region), while it behaves as a long range potential for the smaller scales (ultraviolet region). The scaling properties, however, are exactly the same in the two regions (compare with [BGPS]) and so are the superficial divergences. This means that superficially the ultraviolet Luttinger model behaves as a renormalizable field theory, i.e. has divergences to all orders of the perturbative expansion. In order to show that divergences are in fact absent, one has to use the non-locality of the interaction, and the symmetry properties of the covariance.

Now the problem is the following one: if one tries to use naively the above properties one has to study the estimate of the single Feynman graphs. Unluckily the analyticity of the quantities of physical interest in the couplings is due to some estimates of classes of graphs lumped together. One therefore has to disentangle the classes of graphs enough in order to give a good ultraviolet bound for them, but not too much to loose the good combinatorial behaviour of the estimates. A similar analysis is presented in [LL], but in the present work the decomposition of the graphs is carried out to a much deeper extent. The fundamental result of the present paper is the proof of the analyticity and the exponential decay of the effective potential on scale $p_{0}^{-1}$ obtained by "integrating all the scales" in the ultraviolet region. Elsewhere (see the bibliography, expecially [BGM] and [BGPS]) the infrared problem (physically the more interesting one) of the Luttinger and more realistic models is treated, under the hypothesis that the ultraviolet is also soluble. Therefore our work provides the proof of such an assumption.

The paper is organized as follows: in Sect. 1 we define the Luttinger model and the regularized version of it, and we state rigorously our result; in Sect. 2 we briefly 
present the standard analysis of the superficial divergences of the model, and the absence of factorials in the perturbative expansion (compare again with [BGPS]); in Sects. 3 and 4 we discuss the above mentioned decomposition and we show how it is possible to improve the superficial bounds of the theory, by using the long range decay of the interaction and the symmetry properties of the model. Finally in Sect. 5 we discuss the modifications due to the presence of some additional terms in the interaction.

Finally, let us outline the technical difference between this work and other similar results in constructive quantum field theory, see e.g. [GK, LL, BGPS]. The standard technique used in the above (and in many other) works, is to resum the ultraviolet divergences in the so-called $\beta$-function, and then to study the perturbative convergence of the quantities of physical interest to all orders. This is done by either using an inductive procedure (see [GK, LL]), or computing explicitly the $\beta$-function by means of the tree graphs technique (see [BGPS]). In our case, where we met the same ultraviolet superficial divergences of the above mentioned works, the symmetry properties of the graphs imply that no resummation has to be performed in order to prove the ultraviolet convergence.

To our knowledge there are no other examples of rigorous study of graph symmetry properties in a fermionic theory, not destroying the good combinatorial behaviour of the coefficients of the perturbative expansion. We strongly suspect that it would be difficult to obtain this result without using the tree graphs technique.

Let us remark also that our results are obtained in euclidean space, i.e. for imaginary times, but we introduce a regularization that allows us to remove the cut off in the time direction, so obtaining the positive property of the covariance required by the Osterwalder-Schrader axioms.

\section{The Luttinger Model}

The Luttinger model, that we define following [BGM], is described by the Hamiltonian

$$
H=H_{0}+V_{0}
$$

if

$$
\begin{gathered}
H_{0}=\sum_{\vec{\omega}= \pm 1} \int d x: \psi_{x, \vec{\omega}}^{+}(-i \vec{\omega} \vec{\partial}) \psi_{x, \vec{\omega}}^{-}: \\
V_{0}=\sum_{\vec{\omega}, \vec{\omega}^{\prime}= \pm 1} \int d x d y \lambda(x-y): \psi_{x, \vec{\omega}}^{+} \psi_{x, \vec{\omega}}^{-}:: \psi_{y, \vec{\omega}^{\prime}}^{+} \psi_{y, \vec{\omega}^{\prime}}^{-}:
\end{gathered}
$$

where $x=(t, \vec{x}) \in R^{2}, d x=d t d \vec{x}, \psi^{ \pm}$are creation and annihilation field operators, $\vec{\partial}$ is the derivative with respect to the space variable, $::$ denotes the Wick ordering (obtained by rearranging the order of the product of operators so that the annihilation operators are always to the right and multiplying the new product by the parity sign of the permutation necessary to produce it) with respect to the ground state introduced by filling all the positive energy states in the channel $\vec{\omega}=-1$ and all the negative energy states in the channel $\vec{\omega}=+1$; we can rewrite the interaction as

$$
V_{0}=V_{1}+V_{2}
$$


if we set

$$
\begin{aligned}
V_{1} & =\sum_{\vec{\omega}, \vec{\omega}^{\prime}= \pm 1} \int d x d y \lambda(x-y): \psi_{x, \vec{\omega}}^{+} \psi_{x, \vec{\omega}}^{-} \psi_{y, \vec{\omega}^{\prime}}^{+} \psi_{y, \vec{\omega}^{\prime}}^{-}: \\
V_{2} & =\sum_{\vec{\omega}, \vec{\omega}^{\prime}= \pm 1} \int d x d y \lambda(x-y) g(x-y, \vec{\omega}): \psi_{x, \vec{\omega}}^{+} \psi_{y, \vec{\omega}}^{-}:
\end{aligned}
$$

being $g(x-y, \vec{\omega})$ defined in (1.6).

The potential $\lambda(x)$ is of the form

$$
\lambda(x)=v(\vec{x}) \delta(t),
$$

where $\delta$ is the delta function, and $v(\vec{x})$ is supposed to be smooth and rapidly decaying at $\infty$,

$$
|v(\vec{x})| \leq \bar{\lambda} e^{-p_{0}|\vec{x}|} .
$$

This corresponds to the definition given in [BGM] where we choose, for definiteness, $q_{1}(1)=q_{1}(-1)=q_{2}(1)=q_{2}(-1)=$ const.

We can define the following functional integral (effective potential)

$$
e^{V_{\mathrm{eff}}(\varphi)}=\frac{1}{\mathscr{N}} \int P(d \psi) e^{V(\psi+\varphi)} .
$$

Here $V=-V_{0}, \varphi=\left(\varphi^{+}, \varphi^{-}\right)$and $\psi=\left(\psi^{+}, \psi^{-}\right)$are grassmanian fields; $P(d \psi)$ is the grassmanian gaussian measure of covariance

$$
\begin{aligned}
\int P(d \psi) \psi_{x, \vec{\omega}}^{-} \psi_{y, \vec{\omega}^{\prime}}^{+} & =\delta\left(\vec{\omega}-\vec{\omega}^{\prime}\right) g(x-y, \vec{\omega}) \\
& =\delta\left(\vec{\omega}-\vec{\omega}^{\prime}\right) \int \frac{d k_{0} d \vec{k}}{(2 \pi)^{2}} \frac{e^{-i k x}}{-i k_{0}+\vec{\omega} \vec{k}} \frac{1}{2}\left[e^{-i k_{0} 0^{+}}+e^{-i k_{0} 0^{-}}\right]
\end{aligned}
$$

and $\mathscr{N}$ is a normalization constant.

Equation (1.6) needs some remarks. If we wrote simply

$$
\int \frac{d k_{0} d \vec{k}}{(2 \pi)^{2}} \frac{e^{-i k x}}{-i k_{0}+\vec{\omega} \vec{k}}
$$

we would not have a well defined expression in $(t, \vec{x})=(0, \overrightarrow{0})$. The necessity of introducing the factor

$$
\frac{1}{2}\left[e^{-i k_{0} 0^{+}}+e^{-i k_{0} 0^{-}}\right]
$$

is due to the definition of the interaction term in the Hamiltonian model (in particular to the ordering of the field operators appearing in $V_{0}$ ), as it can be easily seen if we reason in the following way.

We introduce the notation, as in [BG],

$$
\begin{aligned}
& g_{+}\left(\vec{x}-\vec{y}, x_{0}-y_{0}, \vec{\omega}\right)=\left\langle\psi_{\vec{x}, x_{0}, \vec{\omega}}^{-} \psi_{\vec{y}, y_{0}, \vec{\omega}}^{+}\right\rangle, \\
& g_{-}\left(\vec{x}-\vec{y}, x_{0}-y_{0}, \vec{\omega}\right)=\left\langle\psi_{\vec{x}, x_{0}, \vec{\omega}}^{+} \psi_{\vec{y}, y_{0}, \vec{\omega}}\right\rangle,
\end{aligned}
$$

(where $\langle\cdot\rangle$ is the average of $\cdot$ on the ground state at zero temperature) so that we have

$$
\begin{aligned}
g(\vec{x}, t, \vec{\omega}) & =g_{+}(\vec{x}, t, \vec{\omega}) & & \text { if } \quad t>0, \\
& =-g_{-}(-\vec{x},-t, \vec{\omega}) & & \text { if } \quad t<0
\end{aligned}
$$


which (as it is straightforward to check) satisfies the relationship

$$
g_{-}(-x, \vec{\omega})=-g_{+}(x, \vec{\omega}) .
$$

Then the Hamiltonian model described in [BGM] requires

$$
g(\vec{x}, 0, \vec{\omega})=\frac{1}{2}\left[g_{+}\left(\vec{x}, 0^{+}, \vec{\omega}\right)+g_{-}\left(\vec{x}, 0^{-}, \vec{\omega}\right)\right]
$$

since the case $t=0$ can only arise in the anomalous graphs (cf. [BG] for an analogous discussion as far as the model with quadratic dispersion is concerned) in which a contraction occurs between lines emerging from points with the same time index. Disregarding sets of times of measure zero, such contractions can only be obtained by pairing lines representing operators $\psi^{+}, \psi^{-}$in the same $V_{0}$ in the perturbative series; therefore, if we take in account the parity properties of the interaction potential (1.3), we deduce easily (1.7).

From (1.6), (1.7) we have then

$$
g(x, \vec{\omega})=\frac{1}{2 \pi} P\left(\frac{1}{t+i \vec{\omega} \vec{x}}\right),
$$

where $P$ denotes the operation of taking the principal value of the integral with respect to the space variable $\vec{x}$.

The interest in the effective potential (1.5) is due to the fact that it is simply related to the Schwinger functions, [G1, G2].

In order to give a meaning to (1.6), one has to introduce an ultraviolet (UV) and an infrared (IR) cutoff, and then study what happens when the cutoffs are removed.

Let us discuss now the right choice of the cutoffs. In order to achieve the program presented in the introduction one has to put the UV cutoffs on the space and on the time components in an asymmetric way. In fact if we want to relate the perturbative approach with the results of the exact solution we have to take in account the fact that the exact solution does not have any UV time cutoff.

One possible regularization that preserves this feature of the exact solution is therefore the following one.

We write

$$
g(x, \vec{\omega})=g_{\mathrm{IR}}(x, \vec{\omega})+g_{\mathrm{UV}}(x, \vec{\omega})
$$

where

$$
\begin{gathered}
g_{\mathrm{IR}}(x, \vec{\omega})=\int \frac{d k_{0} d \vec{k}}{(2 \pi)^{2}} \frac{e^{-i k x}}{-i k_{0}+\vec{\omega} \vec{k}} e^{-p_{0}^{-2}\left(k_{0}^{2}+\vec{k}^{2}\right)}, \\
g_{\mathrm{UV}}(x, \vec{\omega})=\int \frac{d k_{0} d \vec{k}}{(2 \pi)^{2}} \frac{e^{-\imath k x}}{-i k_{0}+\vec{\omega} \vec{k}}\left(1-e^{-p_{0}^{-2}\left(k_{0}^{2}+\vec{k}^{2}\right)}\right) \frac{1}{2}\left[e^{-i k_{0} 0^{+}}+e^{-i k_{0} 0^{-}}\right] .
\end{gathered}
$$

We decompose $g_{\mathrm{UV}}$ as

$$
g_{\mathrm{UV}}(x, \vec{\omega})=\lim _{N \rightarrow \infty} \sum_{n=0}^{\infty} g_{N}^{(n)}(x, \vec{\omega})
$$

where, if $n>N$,

$$
g_{N}^{(n)}(x, \vec{\omega})=\int \frac{d k_{0} d \vec{k}}{(2 \pi)^{2}} \frac{e^{-i k x}}{-i k_{0}+\vec{\omega} \vec{k}} e^{-\frac{\vec{k}^{2}}{\gamma^{2 N} p_{0}^{2}}}\left(e^{-\frac{k_{0}^{2}+\vec{k}^{2}}{\gamma^{2(n+1)} p_{0}^{2}}}-e^{-\frac{k_{0}^{2}+\vec{k}^{2}}{\gamma^{2 n} p_{0}^{2}}}\right),
$$


and, if $n<N$

$$
\begin{aligned}
g_{N}^{(n)}(x, \vec{\omega})= & \int \frac{d k_{0} d \vec{k}}{(2 \pi)^{2}} \frac{e^{-i k x}}{-i k_{0}+\vec{\omega} \vec{k}}\left(e^{-\frac{k_{0}^{2}+\vec{k}^{2}}{\gamma^{2(n+1)} p_{0}^{2}}}-e^{-\frac{k_{0}^{2}+\vec{k}^{2}}{\gamma^{2 n} p_{0}^{2}}}\right) \\
& +\int \frac{d k_{0} d \vec{k}}{(2 \pi)^{2}} \frac{e^{-\imath k x}}{-i k_{0}+\vec{\omega} \vec{k}}\left(e^{-\frac{\vec{k}^{2}}{\gamma^{2 N} p_{0}^{2}}}-1\right)\left(e^{-\frac{k_{0}^{2}+\vec{k}^{2}}{\gamma^{2(n+1)} p_{0}^{2}}}-e^{-\frac{k_{0}^{2}+\vec{k}^{2}}{\gamma^{2 n} p_{0}^{2}}}\right) \\
\equiv & g^{(n)}(x, \vec{\omega})+\bar{g}_{N}^{(n)}(x, \vec{\omega})
\end{aligned}
$$

Here $\gamma$ is a real number such that $\gamma-1$ is strictly positive.

The covariances defined above have the following properties:

i) if $n>N$

$$
\begin{gathered}
\left|g_{N}^{(n)}(x, \vec{\omega})\right| \leq G_{1} \gamma^{N} e^{-\tilde{\xi}|\vec{x}| \gamma^{N}} e^{-\tilde{\xi}|t| \gamma^{n}} \\
\int_{R^{2}} d x g_{N}^{(n)}(x, \vec{\omega})=0
\end{gathered}
$$

ii) if $n<N$

$$
\begin{gathered}
\left|\bar{g}_{N}^{(n)}(x, \vec{\omega})\right| \leq G_{2} \gamma^{-2 N+3 n} e^{-\tilde{\xi}|\vec{x}| \gamma^{n}} e^{-\tilde{\xi}|t| \gamma^{n}} \\
\int_{R^{2}} d x \bar{g}_{N}^{(n)}(x, \vec{\omega})=0
\end{gathered}
$$

iii) for the covariance $g^{(n)}$, we have

$$
g^{(n)}(x, \vec{\omega})=\frac{\left(\gamma^{n} p_{0}\right)^{2}}{8 \pi} \int_{1}^{\gamma^{2}} d \alpha e^{-\frac{1}{4} \alpha\left(\gamma^{n} p_{0} x\right)^{2}}(t-i \vec{\omega} \vec{x})
$$

with $x^{2}=\vec{x}^{2}+t^{2}$, and therefore we can write

$$
\begin{gathered}
g^{(n)}(x, \vec{\omega})=\gamma^{n} g^{(0)}\left(\gamma^{n} x, \vec{\omega}\right), \\
\left|g^{(0)}(x, \vec{\omega})\right| \leq G_{0} e^{-\xi|\vec{x}|} e^{-\xi|t|}, \\
\int_{R^{2}} d x g^{(n)}(x, \vec{\omega}) \equiv 0,
\end{gathered}
$$

and, due to its symmetry in $t$ and $x$,

$$
\int_{R^{2}} d x g^{(n)}(x, \vec{\omega}) g^{(m)}(x, \vec{\omega}) \equiv 0, \quad \forall n, m,
$$

which is the more striking and remarkable peculiarity of the model.

In (1.13)-(1.16) we can set $\tilde{\xi}=\left(p_{0} / 2\right)\left(1+\gamma^{2}\right)^{-1 / 2}, \xi=p_{0} / 2, G_{1}=\left(p_{0} / \pi\right)\left(\gamma^{2}-1\right)$, $G_{2}=\left(5 p_{0} / 2 \pi\right)\left(\gamma^{4}-1\right)$, and $G_{0}=\left(\gamma^{2}-1\right)\left(p_{0} / \pi\right)=G_{1}$.

If we define $P_{\mathrm{IR}}(d \psi), P_{\mathrm{UV}}(d \psi)$ as the measures of covariance, respectively, $g_{\mathrm{IR}}(x, \vec{\omega}), g_{\mathrm{UV}}(x, \vec{\omega})$ and

$$
e^{V^{(0)}(\varphi)}=\frac{1}{\mathscr{N}} \int P_{\mathrm{UV}}(d \varphi) e^{V(\psi+\varphi)}
$$


we have

$$
e^{V_{\text {eff }}(\varphi)}=\int P_{\mathrm{IR}}(d \psi) e^{V^{(0)}(\psi+\varphi)} .
$$

The (1.19) is the expression we have to consider if we want to resolve the infrared problem; this is what is done in [BGM] under the hypothesis that it is possible to give a meaning to (1.18). In order to do this, we introduce the UV cutoff, by replacing (1.12) with

$$
g_{N}^{(\leq M)}(x, \vec{\omega})=\sum_{n=0}^{M} g_{N}^{(n)}(x, \vec{\omega}), \quad M \gg N
$$

so that

$$
g_{\mathrm{UV}}(x, \vec{\omega})=\lim _{N \rightarrow \infty} \lim _{M \rightarrow \infty} g_{N}^{(\leq M)}(x, \vec{\omega})
$$

and

$$
V^{(0)}(\varphi)=\lim _{N \rightarrow \infty} \lim _{M \rightarrow \infty} \log \frac{1}{\mathscr{N}^{(0)}} \int P_{N}^{(\leq M)}(d \psi) e^{V(\psi+\varphi)}
$$

where $P_{N}^{(\leq M)}(d \psi)$ is the measure of covariance (1.20).

We want to prove the following fundamental result:

Theorem 1.1. The effective potential defined in (1.21) can be written in the following form:

$$
V^{(0)}(\varphi)=\sum_{n} \int d x_{1} \ldots d x_{2 n} V\left(x_{1} \ldots x_{2 n}\right): \varphi_{x_{1}}^{+} \ldots \varphi_{x_{n}}^{+} \varphi_{x_{n+1}}^{-} \ldots \varphi_{x_{2 n}}^{-}:,
$$

where the kernels $V\left(x_{1} \ldots x_{2 n}\right)$ have the following property:

$$
\begin{aligned}
& V\left(\Delta_{1}, \ldots, \Delta_{q}\right) \\
& \left.\quad \equiv \int_{\Delta_{1}} d x_{1}\right|_{\Delta_{2} \times \ldots \times \Delta_{q}} d x_{2} \ldots d x_{q} \int_{R^{2} \times \ldots \times R^{2}} d x_{q+1} \ldots d x_{2 n} V\left(x_{1} \ldots x_{2 n}\right) \mid \\
& \quad \leq \bar{\lambda}^{\delta_{n, 1}} \bar{\lambda}^{n-1} \sum_{m=0}^{\infty}\left(C_{0}^{m} \bar{\lambda}^{m}\right) e^{-\kappa p_{0} d_{(0)}\left(\Delta_{1}, \ldots, \Delta_{q}\right)}
\end{aligned}
$$

i.e. the integrals $V\left(\Delta_{1}, \ldots, \Delta_{q}\right)$ are analytical in $\bar{\lambda}$ and have an exponential decay on scale $p_{0}^{-1}$. In (1.22) $C_{0}$ and $\kappa$ are suitable constants and $d_{(0)}\left(\Delta_{1}, \ldots, \Delta_{q}\right)$ is the sum of the lengths of the bonds on the shortest tree that connects all the squares $\Delta_{1}, \ldots, \Delta_{q}$ of size $\gamma^{-k}$ and $\gamma>1$.

Instead of proving directly this result, we start by proving a complementary result: the analyticity and the exponential decay of $\bar{V}^{(0)}(\varphi)$ defined by

$$
\bar{V}^{(0)(\varphi)}=\lim _{N \rightarrow \infty} \log \frac{1}{\mathscr{N}^{(0)}} \int P^{(\leq N)}(d \psi) e^{V(\psi+\varphi)},
$$

where $P^{(\leq N)}(d \psi)$ is the measure with covariance $g^{(\leq N)}(x, \vec{\omega}) \equiv \sum_{n=0}^{N} g^{(n)}(x, \vec{\omega})$. At the end of Sect. 5 we will discuss the modifications that we have to bring in our proof in order to study the true effective potential with covariance $g_{N}^{(\leq M)}(x, \vec{\omega})$. 
Let us remark here, however, that is quite clear that the study of the (1.23) is the difficult problem: as it is easy to see from (1.13) and (1.14), the covariances $g_{N}^{(n)}$ and $\bar{g}_{N}^{(n)}$ have better scale properties in the ultraviolet region, with respect to the covariance $g^{(n)}$. It will be clear in a moment that the divergences of the theory disappear when one consideres the contribution to the effective potential (1.21) due to the covariances $g_{N}^{(n)}$ and $\bar{g}_{N}^{(n)}$.

\section{The Ultraviolet Problem: Standard Scaling Estimates}

In this section we will study the effective potential (1.23) and we will prove that, except for a class of terms, the results of Theorem 1.1 hold. This is a standard task (see for example [GK]), and we write it in some detail just to introduce the notations and to clarify the strategy we shall follow in the next sections, where the proof of the estimate improvement for the terms of $\bar{V}^{(0)}(\varphi)$ which, in the naive analysis of this section, have divergent bounds will be presented.

In analogy to $\bar{V}^{(0)}(\varphi)$, we can define, on scale $k=0,1,2, \ldots, N-1$,

$$
e^{\bar{V}^{(k)}\left(\psi^{(\leq k)}\right)}=\int P^{(k+1)}(d \psi) \ldots P^{(N)}(d \psi) e^{\bar{V}^{(N)}\left(\psi^{(\leq N)}\right)},
$$

where $e^{\bar{V}^{(N)}\left(\psi^{(\leq N)}\right)}$ is obtained from (1.1), (1.2) by the substitution $\psi \rightarrow \psi^{(\leq N)}$ so that $g \rightarrow g_{\mathrm{IR}}+g^{(\leq N)}$, and $P^{(n)}(d \psi)$ is the measure of covariance $g^{(n)}(x, \vec{\omega})$.

We note that $\lambda(x-y) g^{(\leq N)}(x-y)=\delta\left(x_{0}-y_{0}\right) v(\vec{x}-\vec{y}) g^{(\leq N)}(0, \vec{x}-\vec{y})$.

In order to give explicitly the structure of the effective potential we use now the tree graph technique, with which we must assume the reader is familiar (see [G1, GN] for the technique; see also [BGPS] for a similar construction). A generic term of the effective potential of order $n$ [see (2.1)] can be written as follows:

$$
\bar{V}^{(k)}\left(n, \psi^{(\leq k)}\right)=\int d \underline{x} \sum_{\substack{\gamma: k(\gamma)=k \\ \sigma(\gamma)=n}} \sum_{\left\{h_{v}\right\}} \sum_{\left\{P_{v_{0}}\right\}} V\left(\gamma,\left\{h_{v}\right\}, P_{v_{0}}, \underline{x}\right): \tilde{\psi}^{(\leq k)}\left(P_{v_{0}}\right):
$$

where:

i) the effective potential on scale $k$ is $V^{(k)}\left(\psi^{(\leq k)}\right)=\sum_{n=1}^{\infty} V^{(k)}\left(n, \psi^{(\leq k)}\right)$;

ii) $\sum_{\gamma}$ is the sum on all the trees $\gamma$ of order $\sigma(\gamma)=n$ (i.e. with $n$ end points) and root's frequency $k(\gamma)=k$; e.g.:

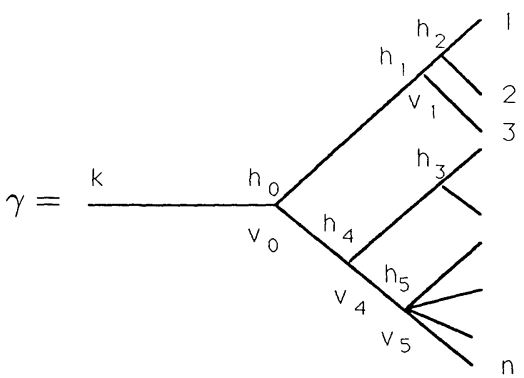


iii) to each end point we associate a graph element among the following ones

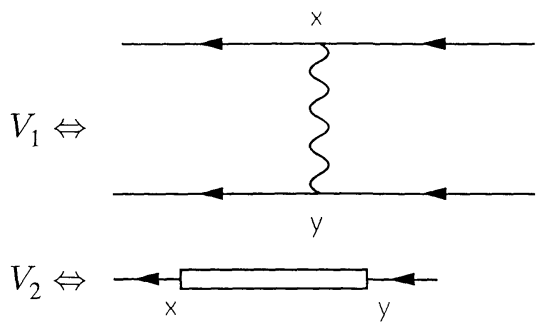

where

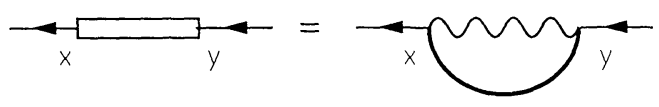

if we define

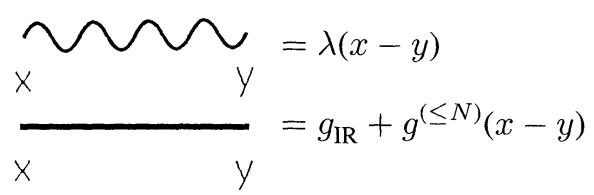

(henceforth we will drop the label $\omega$ foir it turns out to be inessential to our treatment; this means that, in all the following estimates, we always have to sum over the channel indices $\vec{\omega}$ 's, and, to a given perturbative order $n$, the summation yields an extra factor $C^{n}$, with $C=4$ ) so that, if $n_{2}\left[n_{4}\right]$ is the number of end points associated with a two [four] leg graph element, we have the obvious constraint $n_{2}+n_{4}=n$. We assign a label $i=1, \ldots, n_{\gamma}=2 n_{2}+4 n_{4}$ to each field, to distinguish them;

iv) $\underline{x}=\left\{x_{\imath}, y_{\imath}\right\}_{i=1}^{n}$ and $d \underline{x}=\prod_{i=1}^{n} d x_{i} d y_{i}$;

v) $\sum_{\left\{h_{v}\right\}}$ is the sum on all the ways to put the frequencies $h_{v}$ on the tree $\gamma$, increasing along with the tree ordering;

vi) $P_{v_{0}}$ is a subset of $I_{v_{0}}=\left\{1, \ldots, n_{\gamma}\right\}$ and $\sum_{\left\{P_{v_{0}}\right\}}$ is the sum on all such subsets; vii) : $\tilde{\psi}^{(\leq k)}\left(P_{v_{0}}\right):=: \prod_{\imath \in P_{v_{0}}}\left[\psi^{(\leq k)^{\sigma}}\left(z_{i}\right)\right]:$, where $z_{i} \in \underline{x}$ is the coordinate of the field whose label is $i$, and $\sigma_{\imath}= \pm$.

The explicit expression of the kernel $V\left(\gamma,\left\{h_{v}\right\}, P_{v_{0}}, \underline{x}\right)$ is given by

$$
\begin{aligned}
V\left(\gamma,\left\{h_{v}\right\}, P_{v_{0}}, x\right) \\
=\prod_{v \geq v_{0}}\left[\sum_{P_{v_{1}} \ldots P_{v_{s_{v}}}} \sum_{Q_{v}^{(1)} \ldots Q_{v}^{\left(s_{v}\right)}} \mathscr{E}_{\left(<h_{v}\right)}\left(\prod_{j=1}^{s_{v}}: \tilde{\psi}^{\left(<h_{v}\right)}\left(Q_{v}^{(j)} \backslash P_{v} \cap Q_{v}^{(j)}\right):\right)\right. \\
\left.\quad \times \frac{1}{s_{v} !} \mathscr{E}_{\left(h_{v}\right)}^{T}\left(: \tilde{\psi}^{\left(h_{v}\right)}\left(P_{v_{1}} \backslash Q_{v}^{(1)}\right): \ldots,: \tilde{\psi}^{\left(h_{v}\right)}\left(P_{v_{s_{v}}} \backslash Q_{v}^{\left(s_{v}\right)}\right):\right)\right] \\
\quad \times \prod_{j=1}^{n_{4}} \lambda\left(x_{j}-y_{\jmath}\right) \prod_{i=n_{4}+1}^{n}\left(\lambda g_{\mathrm{IR}}+\lambda g^{(\leq N)}\right)\left(x_{i}-y_{i}\right),
\end{aligned}
$$

where: 
i) $s_{v}$ is the number of subtrees $\gamma_{j}^{(v)}$ directly connected to the vertex $v$;

ii) $P_{v_{j}}$ is a subset of the set $I_{v_{j}}$ of the $n_{\gamma_{j}^{(v)}}$ fields at the end of $\gamma_{j}^{(v)}$;

iii) $v_{1}, \ldots, v_{s_{v}}$ are the vertices on higher frequencies directly connected with $v$;

iv) between the sets $\left\{P_{v_{j}}\right\}_{j=1}^{s_{v}},\left\{Q_{v}^{(j)}\right\}_{j=1}^{s_{v}}, P_{v}$, the following inclusion relations are imposed:

$$
P_{v} \subset \bigcup_{j=1}^{s_{v}} Q_{v}^{(\jmath)}, \quad Q_{v}^{(j)} \subset P_{v_{\jmath}}, \quad P_{v_{0}} \cap I_{v} \subset P_{v}
$$

v) when $v$ is such that, for some $j=1, \ldots, s_{v}, v_{j}$ is an end point of the tree, then the sum on $P_{v_{j}}$ has only one addend, i.e. $P_{v_{\jmath}} \equiv I_{v_{\jmath}} \equiv$ the set of fields appearing in the corresponding graph element;

vi) we have labeled, for simplicity, the points $\left\{x_{i} y_{\imath}\right\}_{i=1}^{n}$ so that the first $n_{4}$ pairs are of $\lambda$-type, and the last $n_{2}$ pairs are of $\lambda g^{(\leq N)}$-type;

vii) $\mathscr{E}_{(<h)}$ is the expectation value (simple expectation) with respect to the measure $P\left(d \psi^{(<h)}\right.$;

viii) $\mathscr{E}_{(h)}^{T}$ is the truncated expectation on scale $h$, as it is defined in [G1].

In the derivation of the (2.3) we used the following properties of the Wick product. Because of the independence of the fields on different scale, we can write

$$
: \tilde{\psi}^{(\leq h)}(P):=\sum_{Q \subset P}(-1)^{\pi}: \tilde{\psi}^{(<h)}(Q):: \tilde{\psi}^{(h)}(P \backslash Q):
$$

where $\pi$ is a parity which depends on the ordering of the fields with respect to the original ordering, and which we write explicitly neither here nor later, in analogous situations [see, e.g., (2.8)], for it turns to be inessential.

Moreover

$$
\prod_{j=1}^{s}: \tilde{\psi}\left(Q_{j}\right):=\sum_{P \subset \bigcup_{j=1}^{s} Q_{j}}(-1)^{\pi}: \tilde{\psi}(P): \mathscr{E}\left(\prod_{j=1}^{s}: \tilde{\psi}\left(Q_{j} \backslash P \cap Q_{j}\right):\right)
$$

where $\mathscr{E}(\cdot)$ is the expecation value of - with respect to the measure defined by the Wick ordering.

We want to show now that, defining

$$
V\left(n, \gamma, P_{v_{0}}\right)=\left.\int_{\Delta_{1}} d x_{1}\right|_{\substack{\left\{x_{i}^{\prime}\right\}_{\imath=2}^{\left|P_{v_{0}}\right|} \in \Delta_{2} \times \ldots \times \Delta_{\left|P_{v_{0}}\right|} \\ \int \underline{x}^{\prime}}} V\left(\gamma,\left\{h_{v}\right\}, P_{v_{0}}, \underline{x}\right) \mid
$$

we shall have, $\forall n, \gamma, P_{v_{0}}$,

$$
V\left(n, \gamma, P_{v_{0}}\right) \leq C_{0}^{n} e^{-\frac{\bar{\xi}}{2} d_{(k)}\left(\Delta_{1}, \ldots, \Delta_{P_{v_{0}}}\right)} .
$$

In (2.6) we have
i) $\underline{x}^{\prime}=\underline{x} \backslash x_{1}, d \underline{x}^{\prime}=\prod_{i=2}^{n} d x_{i} \prod_{i=1}^{n} d y_{i}$;
ii) $\left\{x_{i}^{\prime}\right\}_{\imath=2}^{\left|P_{v_{0}}\right|} \equiv\left\{x_{i}\right\}_{i=1}^{\left|P_{v_{0}}\right|} \backslash x_{1}$;
iii) $\bar{\xi}$ is to be fixed later [see (2.20)]; 
iv) $d_{(k)}\left(P_{v_{0}}\right)$ is constructed in the following way: let us call $\mu$ the shortest tree that connects all the points labeled by $P_{v_{0}}$ (note that $\gamma$ and $\mu$ are trees with a very different meaning!), and let us call $\lambda^{(1)}, \ldots, \lambda^{\left(\left|P_{v_{0}}\right|-1\right)}$ the bonds of $\mu$; let us call moreover $\lambda_{i}^{(j)}, i=0,1$, the two components of $\lambda^{(j)}$, so that $\lambda^{(j)}=\left(\lambda_{0}^{(j)}, \lambda_{1}^{(j)}\right)$, being the first one the time component and the second one the space component; then

$$
d_{(k)}\left(P_{v_{0}}\right)=\sum_{j=1}^{\left|P_{v_{0}}\right|-1}\left(\gamma^{k}\left|\lambda_{0}^{(\jmath)}\right|+\left|\lambda_{1}^{(\jmath)}\right|\right) ;
$$

v) $\Delta_{1}, \ldots, \Delta_{\left|P_{v_{0}}\right|}$ are square subsets of $R^{2}$ of side $\gamma^{-k}$ (not necessarily all distinct) which are contained in a pavement of $R^{2}$;

vi) $d_{(k)}\left(\Delta_{1}, \ldots, \Delta_{\left|P_{v_{0}}\right|}\right)$ is the minimum of $d_{(k)}\left(P_{v_{0}}\right)$ defined by (2.8) on the domain of definition of the variables of the fields in $P_{v_{0}}$.

All the rest of the paper is devoted to the proof of the bound (2.7). We want to stress that the (2.7) is one possible way to formulate the properties of $V_{\mathrm{eff}}^{(0)}$ that are assumed in [BGM].

In order to prove the bound (2.7) we shall use the following fundamental results for the fermionic simple expectations and truncated expectations (see [GK, LL]; see also Appendix A4 for the generalization of the proof to the case with the Wick ordering)

$$
\begin{gathered}
\left.\mid \mathscr{E}_{(h)}\left(\prod_{j}: \tilde{\psi}^{(h)}\left(P_{j}\right):\right)\right) \mid \leq \gamma^{\frac{h}{2} \sum_{\jmath}\left|P_{\jmath}\right|} C_{1}^{\sum_{j}\left|P_{\jmath}\right|}, \\
\left.\mid \mathscr{E}_{(<h)}\left(\prod_{j}: \tilde{\psi}^{(<h)}\left(P_{\jmath}\right):\right)\right) \mid \leq \gamma^{\frac{h}{2} \sum_{\jmath}\left|P_{j}\right|} C_{2}^{\sum_{j}\left|P_{j}\right|}
\end{gathered}
$$

with $C_{2}>C_{1}$, and

$$
\begin{aligned}
& \left|\mathscr{E}_{(h)}^{T}\left(: \tilde{\psi}^{(h)}\left(P_{1}\right):, \ldots,: \tilde{\psi}^{(h)}\left(P_{s}\right):\right)\right| \\
& \quad \leq \gamma^{\frac{h}{2} \sum_{\jmath}\left|P_{\jmath}\right|} C_{3}^{\sum_{j}\left|P_{\jmath}\right|} \sum_{\mu} e^{-\xi d_{\mu(h)}\left(P_{1}, \ldots, P_{s}\right)},
\end{aligned}
$$

where $d_{\mu(h)}$ is constructed in the following way: let $\mu$ be a tree graph between the clusters of points labeled by $P_{1}, \ldots, P_{s}$ such that the clusters are joined in a connected way and let $\sum_{\mu}$ be the sum of such trees; let $\lambda^{(1)}, \ldots, \lambda^{(s-1)}$ be the bonds of the tree; let us write $\lambda^{(\jmath)}=\left(\lambda_{0}^{(\jmath)}, \lambda_{1}^{(\jmath)}\right)$; then

$$
d_{\mu(h)}\left(P_{1}, \ldots, P_{s}\right)=\sum_{j=1}^{s-1} \gamma^{h}\left(\left|\lambda_{0}^{(j)}\right|+\left|\lambda_{1}^{(j)}\right|\right) .
$$

In order to avoid convergence problems due to the $V_{2}$ term of the interaction we need some preliminary rewriting of the simple and truncated expectations. We recall that, once we have fixed the sets

$$
\left\{\left\{P_{v_{j}}\right\}_{j=1}^{s_{v}},\left\{Q_{v}^{(j)}\right\}_{j=1}^{s_{v}}\right\}_{v \geq v_{0}}
$$

which we will sum over in a latter time, to each end point of type $V_{2}$ there correspond two fields. Each non-external field will be contracted on a fixed scale, say $h_{V}$ if the 
field is contained in the set

$$
\bigcup_{j=1}^{s_{V}} P_{V_{j}} \backslash Q_{V}^{(j)}
$$

or in the set

$$
\bigcup_{j=1}^{s_{V}} Q_{V}^{(j)} \backslash P_{V} \cap Q_{V}^{(j)}
$$

Then, if $\psi_{y_{\imath}}^{\sigma_{\imath}}$ is the field which will be contracted on the lowest scale, we can write

$$
\psi_{t_{\imath}, \vec{y}_{i}}^{\sigma_{\imath}}=\psi_{t_{\imath}, \vec{x}_{i}}^{\sigma_{2}}+\int_{0}^{1} d \alpha\left[\left.\partial_{\vec{z}_{\imath}} \psi_{t_{\imath}, \vec{z}_{i}}^{\sigma_{\imath}}\right|_{\vec{z}_{i}=\vec{x}_{i}+\alpha\left(\vec{y}_{i}-\vec{x}_{i}\right)}\right]\left(\vec{y}_{\imath}-\vec{x}_{i}\right),
$$

and therefore we can rewrite the expectation whose argument contains the field $\psi_{y_{\imath}}^{\sigma_{\imath}}$ as sum over two terms.

Now, $\forall i=n_{4}+1, \ldots, n$, we decompose

$$
\begin{aligned}
\left(\lambda g^{(\leq N)}\right)\left(x_{i}-y_{\imath}\right) & \equiv\left(\lambda g^{(\leq N)}\right)\left(0, \vec{x}_{\imath}-\vec{y}_{\imath}\right) \\
& =\left(\lambda g^{\leq h)}\right)\left(0, \vec{x}_{i}-\vec{y}_{i}\right)+\left(\lambda g^{(h+1, N)}\right)\left(0, \vec{x}_{\imath}-\vec{y}_{i}\right)
\end{aligned}
$$

where $h$ is the frequency associated to the vertex to which the considered point is connected directly (it is $h \geq h_{V}$ ), and we have defined

$$
g^{(n, m)}(x, \omega)=\sum_{k=n}^{m} g^{(n)}(x, \omega) .
$$

So, for each end point of type $V_{2}$ we have two contributions, corresponding to the two terms of the decomposition (2.15), and we want to study them separately.

If we write the expression (2.3), and use the decomposition (2.15) and the bounds (2.10), (2.11), we have

$$
\begin{aligned}
& \left|V\left(\gamma,\left\{h_{v}\right\}, P_{v_{0}}, x\right)\right| \\
& \leq \prod_{v \geq v_{0}} \sum_{P_{v_{1}} \ldots P_{v_{s}}} \sum_{Q_{v}^{(1)} \ldots Q_{v}^{(s)}} \prod_{v \geq v_{0}} \gamma^{\frac{h_{v}}{2}\left(\sum_{\jmath}\left|P_{v_{j}}\right|-\left|P_{v}\right|\right)} C_{0}^{\sum_{j}\left|P_{v_{j}}\right|-\left|P_{v}\right|} \\
& \quad \times \prod_{i=n_{4}+1}^{n} \mid\left(\lambda g_{\mathrm{IR}}\right)\left(0, \vec{x}_{i}-\vec{y}_{\imath}\right)+\left(\lambda g^{(\leq h)}\right)\left(0, \vec{x}_{i}-\vec{y}_{\imath}\right) \\
& \quad+\left(\lambda g^{(h+1, N)}\right)\left(0, \vec{x}_{i}-\vec{y}_{\imath}\right) \mid \\
& \quad \times \prod_{j=1}^{n_{4}}\left|\lambda\left(x_{j}-y_{j}\right)\right| \frac{1}{s_{v} !} \sum_{\mu} e^{-\xi d_{\mu\left(h_{v}\right)}\left(P_{v_{1}}, \ldots, P_{v_{s_{v}}}\right)}
\end{aligned}
$$

where $C_{0}=\max \left\{C_{2}, C_{3}\right\}$. If we want to obtain now the bound (2.7) we have to integrate the expression (2.17). It is evident that only the last three lines of (2.17) 
depend on $\underline{x}$; therefore we must evaluate the following expression:

$$
\begin{aligned}
& \quad \int d \underline{x} \frac{1}{s_{v} !} \sum_{\mu} e^{-\xi d_{\mu(h v)}\left(P_{v_{1}}, \ldots, P_{v_{s}}\right)} \prod_{\jmath=1}^{n_{4}}\left|\lambda\left(x_{\jmath}-y_{\jmath}\right)\right| \\
& \left\{x_{\imath}\right\}_{\imath=1}^{P_{v_{0}} \in \Delta_{1} \times \ldots \times \Delta_{P_{v_{0}}}} \\
& \quad \times \prod_{\imath=n_{4}+1}^{n} \mid\left(\lambda g_{\mathrm{IR}}\right)\left(0, \vec{x}_{\imath}-\vec{y}_{\imath}\right)+\left(\lambda g^{(\leq h)}\right)\left(0, \vec{x}_{\imath}-\vec{y}_{\imath}\right) \\
& +\left(\lambda g^{(h+1, N)}\right)\left(0, \vec{x}_{i}-\vec{y}_{i}\right) \mid .
\end{aligned}
$$

The estimate of (2.18) is standard, [G1], but we have to treat carefully the terms due to the decomposition (2.15).

If we treat them separately, it is straightforward to realize that, each time we have a term $\lambda g^{(h+1, N)}$, the decomposition of the expectation implied by (2.14) gives two contributions: the first one is vanishing for parity reasons, the second one gives a factor

$$
C \gamma^{-h} \gamma^{h_{V}} \leq C
$$

because $h_{V} \leq h$. The factor $\lambda g_{\mathrm{IR}}$ is integrable and gives a constant which can be lumped together with (2.19). Each time we have a factor $\lambda g^{(\leq h)}$ we obtain a factor $h$ so that we can conclude that $(2.18)$ can be bounded by

$$
\gamma^{-2 k} \mathscr{C}^{n} \bar{\lambda}^{n} e^{-\frac{\bar{\xi}}{2} d_{(k)}\left(\Delta_{1}, \ldots, \Delta_{\left|P v_{0}\right|}\right)} \prod_{\substack{v \geq v_{0} \\ d_{2}(v)=0}} h_{v}^{n_{2}, v} \prod_{v \geq v_{0}} \gamma^{-2 h_{v}\left(s_{v}-1\right)}
$$

where $\bar{\xi}=\min \left\{\xi, p_{0}\right\}$, and where, given a vertex $v$, we define $m(v)$ the number of vertices we can meet moving forward along one direction from the considered vertex $v$ up to some end point, and we set $d_{1}(v)=\max \{m(v)\}$ and $d_{2}(v)=\min \{m(v)\}$. We call $d_{1}(v)$ the degree of the vertex $v$.

Then we have

$$
\begin{aligned}
V\left(n, \gamma, P_{v_{0}}\right) \leq & \mathscr{C}^{n} \bar{\lambda}^{n} e^{-\frac{\bar{\xi}}{2} d_{(k)}\left(\Delta_{1}, \ldots, \Delta_{\left|P_{v_{0}}\right|}\right)} \sum_{\left\{h_{v}\right\}} \prod_{\substack{v \geq v_{0} \\
d_{2}(v)=0}} h_{v}^{n_{2, v}} \\
& \times \prod_{v \geq v_{0}} \sum_{P_{v_{1}} \ldots P_{v_{s}}} \sum_{Q_{v}^{(1)} \ldots Q_{v}^{(s)}} \gamma^{\frac{h_{v}}{2}\left(\sum_{j}\left|P_{v_{j}}\right|-\left|P_{v}\right|\right)} \gamma^{-2 h_{v}\left(s_{v}-1\right)} .
\end{aligned}
$$

We shall use now the following identities

$$
\begin{aligned}
\sum_{v \geq v_{0}} h_{v}\left(s_{v}-1\right)= & \sum_{v \geq v_{0}}\left(h_{v}-h_{v^{\prime}}\right)\left(n_{v}-1\right)+k(n-1), \\
\sum_{v \geq v_{0}} h_{v}\left(\sum_{j}\left|P_{v_{j}}\right|-\left|P_{v}\right|\right)= & \sum_{v \geq v_{0}}\left(h_{v}-h_{v^{\prime}}\right)\left(4 n_{4, v}+2 n_{2, v}-\left|P_{v}\right|\right) \\
& +k\left(4 n_{4}+2 n_{2}-\left|P_{v_{0}}\right|\right), \\
\sum_{\substack{v \geq v_{0} \\
d_{2}(v)=0}} h_{v} n_{2, v}= & \sum_{v \geq v_{0}}\left(h_{v}-h_{v^{\prime}}\right) n_{2, v}+k n_{2},
\end{aligned}
$$


and the fact that

$$
h \leq C \gamma^{\varepsilon h} \quad \forall h
$$

for $\varepsilon>0$ and $C=(e \varepsilon \log \gamma)^{-1}$. So we have, redefining the constant $\mathscr{C}$,

$$
\begin{aligned}
V\left(n, \gamma, P_{v_{0}}\right) \leq & \mathscr{C}^{n} \bar{\lambda}^{n} \gamma^{-\frac{k}{2} \alpha_{v_{0}}} e^{-\frac{\bar{\xi}}{2} d_{(k)}\left(\Delta_{1}, \ldots, \Delta_{\left|P_{v_{0}}\right|}\right)} \\
& \times \sum_{\left\{h_{v}\right\}} \prod_{v \geq v_{0}}\left\{\sum_{P_{v_{1}} \ldots P_{v_{s_{v}}}} \sum_{Q_{v}^{(1)} \ldots Q_{v}^{(s v)}} \gamma^{-\frac{1}{2} \alpha_{v}\left(h_{v}-h_{v^{\prime}}\right)}\right\}
\end{aligned}
$$

where

$$
\alpha_{v}=\left|P_{v}\right|+2 n_{2, v}(1-\varepsilon)-4 .
$$

If it was $\alpha_{v}>0$ always, it would be sufficient to prove that no factorials arise from the multiple sums in (2.25). Note that in the estimate of such sums we can extract from the exponential in (2.25) a factor $\gamma^{-\frac{1}{2}\left|P_{v}\right|}$, since we have

$$
\prod_{v \geq v_{0}} \gamma^{-\frac{\alpha_{v}}{2}\left(h_{v}-h_{v^{\prime}}\right)}=\gamma^{-\sum_{v \geq v_{0}} \frac{\alpha_{v}}{2}\left(h_{v}-h_{v^{\prime}}\right)},
$$

where each sum starts from 1 because $h_{v} \geq h_{v^{\prime}}+1$. Therefore the bound that we have to prove is the following one:

$$
\prod_{v \geq v_{0}}\left\{\sum_{p_{v_{1}} \ldots P_{v_{s}}} \sum_{Q_{v}^{(1)} \ldots Q_{v}^{\left(s_{v}\right)}} \gamma^{-\frac{1}{2}\left|P_{v}\right|}\right\} \leq C_{4}^{n} .
$$

As a matter of fact, $\alpha_{v}$ is not always strictly positive, but, as we shall see later, it is possible, with a more careful estimate, to replace $\alpha_{v}$ with a new factor $\alpha_{v}^{\prime}$ which is strictly positive for every $P_{v}$,

$$
\alpha_{v} \rightarrow \alpha_{v}^{\prime}>0
$$

Therefore we have two results to prove in order to have the bound (2.7): 1) to show that the substitution (2.28) is possible; 2 ) to verify the bound (2.27).

To the first problem the following sections are devoted; the proof of (2.27) can be found in Appendix A1, and is based on a similar proof in [CNPS].

If we look now at (2.26), we see that $\alpha_{v}>0$ except in the following cases:

i) $\left|P_{v}\right|=2, n_{2, v}=0 \Rightarrow \alpha_{v}=-2<0$,

ii) $\left|P_{v}\right|=2, n_{2, v}=1 \Rightarrow \alpha_{v}=-2 \varepsilon<0$,

iii) $\left|P_{v}\right|=4, n_{2, v}=0 \Rightarrow \alpha_{v}=0$.

We claim that a more careful study enables us to write an expression like (2.25) with the substitution (2.28), where $\alpha_{v}^{\prime}$ is defined by

$$
\alpha_{v}^{\prime}=\alpha_{v}+\left(1-\eta_{1}\right) \delta_{\left|P_{v}\right|, 4} \delta_{n_{2, v}, 0}+\left(2-\eta_{2}\right) \delta_{\left|P_{v}\right|, 2} \delta_{n_{2, v}, 1}+\left(3-\eta_{3}\right) \delta_{\left|P_{v}\right|, 2} \delta_{n_{2, v}, 0},
$$

so that the three above cases give:

i) $\left|P_{v}\right|=2, n_{2, v}=0 \Rightarrow \alpha_{v}^{\prime} \geq(1-\eta)>0$,

ii) $\left|P_{v}\right|=2, n_{2, v}=1 \Rightarrow \alpha_{v}^{\prime} \geq(2-\eta)>0$, 
iii) $\left|P_{v}\right|=2, n_{2, v}=0 \Rightarrow \alpha_{v}^{\prime} \geq(1-\eta)>0$, where $\eta=\max \left\{\eta_{1}, \eta_{2}+2 \varepsilon, \eta_{3}\right\}$ is sufficiently small.

The proof of (2.28), (2.29) is a rather cumbersome treatment, that we divide into two parts: in Sect. 3 we consider what happens when we try to define the effective potential on scale $N-1$, starting with a cutoff on scale $N$; this first case contains the basic features of the general one but it is a little bit simpler. In Sect. 4 we generalize the results to the case of an effective potential on a scale $k<N$, arbitrary.

\section{Renormalization Group: The First Step}

If we restrict ourselves to study the effective potential on scale $k=N-1$, we have only trees of the form

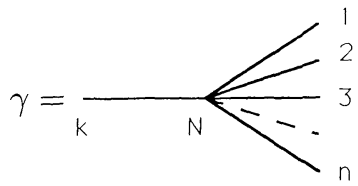

whose corresponding graphs can be symbolically represented by

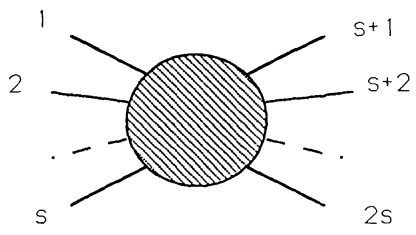

if $\left|P_{v_{0}}\right|=2 s$ is the number of external legs. In such a situation the kernel (2.3) is reduced to a single term (for $v=v_{0}$ ). Henceforth we shall use the following graphic representation for the single expectations and truncated expectations:

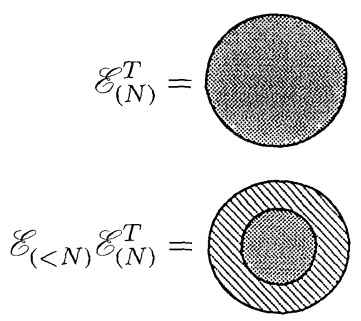

so that the four and two external leg contributions to the effective potential in (2.3) can be thought to have the following structure:
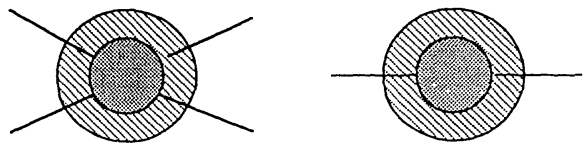

The superficial bounds of Sect. 2 imply a factor $\alpha_{v}$ equal, respectively, to 0 and 2 [or $-2 \varepsilon$ ]. We want to improve the estimate, by using the formula, [LL], that will be given now. 
Theorem 3.1. If we have $k$ clusters $1, \ldots, k$ with $1=x_{1} \cup y_{1}$, then we have, for the truncated expecations on an arbitrary scale $h$,

$$
\begin{aligned}
& \mathscr{E}_{(h)}^{T}\left(\tilde{\psi}\left(x_{1} \cup y_{1}\right), \tilde{\psi}(2), \ldots, \tilde{\psi}(k)\right) \\
& =\mathscr{E}_{(h)}^{T}\left(\tilde{\psi}\left(x_{1}\right), \tilde{\psi}\left(y_{1}\right), \tilde{\psi}(2), \ldots, \tilde{\psi}(k)\right) \\
& +\sum_{K_{1}, K_{2}: K_{1} \cap K_{2}=\emptyset}(-1)^{\pi} \mathscr{E}_{(h)}^{T}\left(\tilde{\psi}\left(x_{1}\right), \tilde{\psi}\left(J_{1}\right), \ldots, \tilde{\psi}\left(J_{\left|K_{1}\right|}\right)\right) \\
& \quad K_{1} \cup K_{2}=\{2, \ldots, k\}=\left\{J_{2}\right\}_{i=1}^{\left|K_{1}\right|+\left|K_{2}\right|} \\
& \left.\quad \times \mathscr{E}_{(h)}^{T}\left(\tilde{\psi} y_{1}\right), \tilde{\psi}\left(J_{\left|K_{1}\right|+1}\right), \ldots, \tilde{\psi}\left(J_{\left|K_{1}\right|+\left|K_{2}\right|}\right)\right),
\end{aligned}
$$

and if we consider the Wick ordering, (3.1) holds still with the constraint that in the truncated expectation at the above second line $\tilde{\psi}\left(x_{1}\right)$ and $\tilde{\psi}\left(y_{1}\right)$ cannot be directly connelted. Note that the sum on $K_{1}, K_{2}$ is bounded by $C^{k}$.

Now we apply the result (3.1) to the three cases discussed at the end of Sect. 2. We organize the discussion as follows.

Let us start showing that the bound of the terms with $\left|P_{v_{0}}\right|=2$ can be improved in the following way:

$$
C \gamma^{N} \rightarrow C N^{2} \leq C \gamma^{2 \varepsilon_{0} N}
$$

with $\varepsilon_{0} \ll 1$ and the constant $C$ depending on $n$ at most as $C_{0}^{n}$. We sketch briefly how the above bound is obtained. The idea is to use the topological properties of the graphs; because of cancellations due to the oddness of the covariance, we can write, provided there is at least one graph element of type $V_{1}$ (see the beginning of Sect.2), the following decomposition

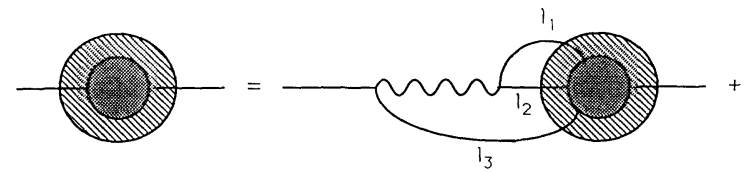

(a)

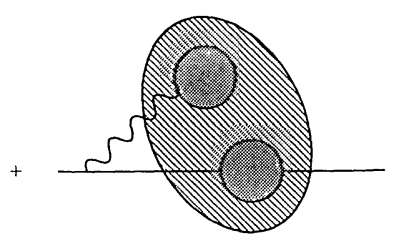

(b)

where, in the contribution (a) at least one line among $l_{1}, l_{2}, l_{3}$ must be on scale $N$ (hard line), and the others can be indifferently on scale $N$ or on scale $<N$ (soft lines) and shall be denoted $g$. In the following, for the sake of brevity, we shall avoid repeating the (evident) conditions for the "hard connectedness" of the graphs, due to the definition of the $\mathscr{E}_{(h)}^{T}$.

Now we use, in order to assure the connection of the graph, a line $g$ instead of a line $\lambda$, and we replace a contribution

$$
\|v\|_{1}\|g\|_{\infty} \leq \bar{\lambda} \gamma^{N}
$$

with a contribution

$$
\|v\|_{\infty}\left\|g\left(t_{1}-t_{2}, \cdot\right)\right\|_{1} \leq \bar{\lambda} N \quad \forall t_{1}-t_{2}
$$


where the factor $N$ appears when the line $g$ is soft, and the $L_{1}$-norm is evaluated on $g^{(h)}$ and then summed on $h=0, \ldots, N$. In order to obtain this second estimate, the integration over a variable $t_{\imath}, i \in\{1,2\}$, appearing in (3.5) must be performed using the decay of another line.

Let us show how this argument goes into the term (a) of (3.3) where we suppose that the line $l_{3}$ is hard (otherwise the discussion is identical, up to a relabeling of the integration variables); we have

$$
\begin{aligned}
& \mid \int d y_{1} d w_{2} d w_{3} d w_{4} d z g_{l_{1}}\left(x_{1}-w_{4}\right) \lambda\left(x_{1}-y_{1}\right) g_{l_{2}}\left(y_{1}-w_{2}\right) g_{l_{3}}\left(y_{1}-w_{3}\right) \\
& \quad \times \mathscr{B}\left(w_{2}-z, w_{3}-z, w_{4}-z\right) \mid \\
& \leq\|v\|_{\infty}\|\mathscr{B}\| \int d\left(\vec{x}_{1}-\vec{w}_{4}\right)\left|g_{l_{1}}\left(t_{1}-t_{4}, \vec{x}_{1}-\vec{w}_{4}\right)\right| \\
& \quad \times \int d\left(\vec{y}_{1}-\vec{w}_{2}\right)\left|g_{l_{2}}\left(t_{1}-t_{2}, \vec{y}_{1}-\vec{w}_{2}\right)\right| \int d\left(t_{1}-t_{3}\right)\left|g_{l_{3}}\left(t_{1}-t_{3}, \vec{y}_{1}-\vec{w}_{3}\right)\right| \\
& \leq\|v\|_{\infty}\|\mathscr{B}\| N^{2} \int d \vec{z}_{1} e^{-\kappa\left|\vec{z}_{1}\right|} \int d \vec{z}_{2} e^{-\kappa\left|\vec{z}_{2}\right|} \int d t_{3} e^{-\kappa\left|t_{3}\right|} \\
& \leq \bar{\lambda}\|\mathscr{B}\| N^{2},
\end{aligned}
$$

where

$$
\|\mathscr{B}\| \equiv \int d x_{1} \int d x_{2} \int d x_{3}\left|\mathscr{B}\left(x_{1}, x_{2}, x_{3}\right)\right| \leq \bar{\lambda}^{n-1} C
$$

if $n$ is the total number of clusters inside the graph, so that we obtain (3.2). In a similar way we treat the case (b) of (3.3); here we have two distinct connected sets, but to avoid the vanishing of the graph there must be a soft line connecting the two sets, and therefore we can integrate on this line instead of the ondulated line drawn in the figure. The number of ways to choose such a line is bounded by $C^{n}$, so that we have no combinatorial problem. In fact, if we call $I_{1}, I_{2}$ the clusters of soft semilines coming out from the two considered sets, and $P_{1} \subset I_{1}$ the subcluster of lines in $I_{1}$ which are not connected to each other, and $P_{2} \subset I_{2}$ the analogous subcluster of lines in $I_{2}$, a line $g^{(<N)}$ will connect a point $w_{1} \in P_{1}$ with a point $w_{2} \in P_{2}$ in such a way that one has for the simple expectations

$$
\mathscr{E}_{(<N)}\left(I_{1} \cup I_{2}\right)=\sum_{P_{1} \subset I_{1}} \sum_{P_{2} \subset I_{2}} \mathscr{E}_{(<N)}\left(I_{1} \backslash P_{1}\right) \mathscr{E}_{(<N)}\left(I_{2} \backslash P_{2}\right) \mathscr{E}_{(<N)}\left(: P_{1}:: P_{2}:\right),
$$

where

$$
\mathscr{E}_{(<N)}\left(: P_{1}:: P_{2}:\right)=\sum_{w_{1} \in P_{1}} \sum_{w_{2} \in P_{2}} g^{(<N)}\left(w_{1}-w_{2}\right) \mathscr{E}_{(<N)}\left(: P_{1} \backslash w_{1}:: P_{2} \backslash w_{2}:\right),
$$

and we can see then that the total number of choices is bounded by

$$
\sum_{p_{1}, p_{2}}\left(\begin{array}{c}
\left|I_{1}\right| \\
p_{1}
\end{array}\right)\left(\begin{array}{c}
\left|I_{2}\right| \\
p_{2}
\end{array}\right)\left(\left|I_{1}\right|-p_{1}\right)\left(\left|I_{2}\right|-p_{2}\right) \leq C^{n}
$$

Now, as in the previous case, we can replace a contribution

$$
\|v\|_{1}\left\|g^{(<N)}\right\|_{\infty} \leq \bar{\lambda} \gamma^{N}
$$


with a contribution

$$
\|v\|_{\infty}\left\|g^{(<N)}(t, \cdot)\right\|_{1} \leq \bar{\lambda} N
$$

so that (3.2) follows. Q.E.D.

This is tantamount to replace, in $(2.3)$, the expression $\mathscr{E}_{(<N)}(\ldots) \mathscr{E}_{(N)}^{T}(\ldots)$ with a new expression, say $A(\ldots)$, such that, if we introduce (2.3) so modified in (2.6), we obtain (2.25), with the following substitution

$$
\alpha_{v_{0}} \rightarrow \alpha_{v_{0}}+2\left(1-\varepsilon_{0}\right) \delta_{\left|P_{v_{0}}\right|, 2}
$$

With this result we treat now the case $\left|P_{v_{0}}\right|=4$. We write here the complete decomposition of the expectations in this case, which is obtained by applying iteratively Theorem 3.1

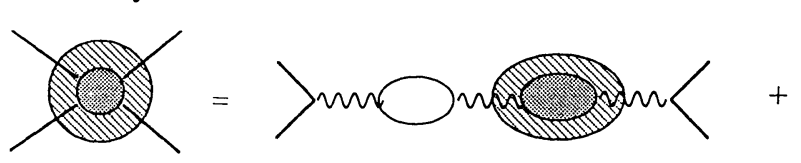

(a)

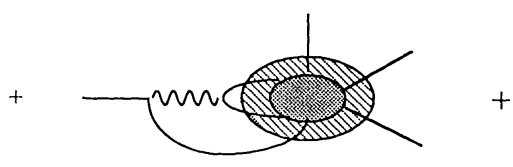

(c)

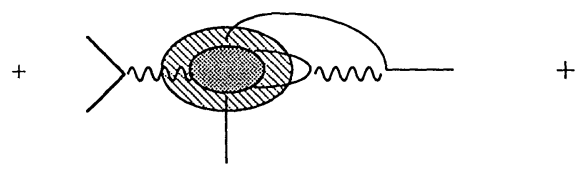

(e)

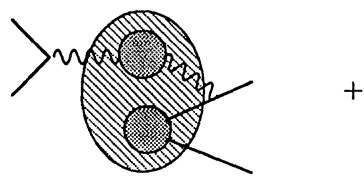

(g)

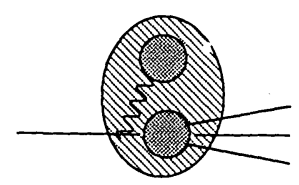

(b)

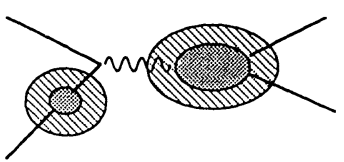

(d)

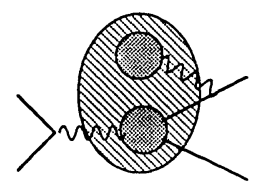

(f)

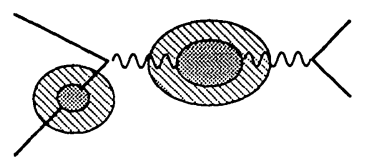

(n)

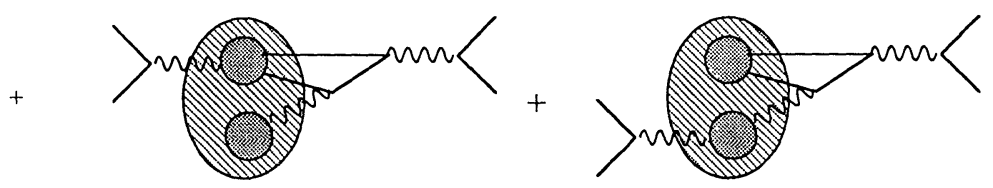

(1)

(j)

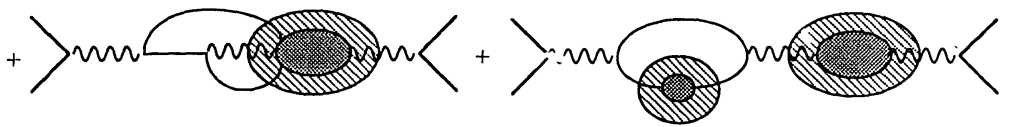

(k) 
where all the contributions but (a), can be bounded, using the argument discussed above [see (3.4), (3.5) and (3.6)], by

$$
C N^{2} \gamma^{-N} \leq C \gamma^{-N\left(1-2 \varepsilon_{0}\right)}
$$

We do not present the detailed analysis of these bounds, for, once the discussion after (3.2) is understood, it's very easy to obtain (3.8). For the same reason, in the following part of the section we shall give only the results, as far as bounds of classes of graphs are concerned. We will omit also the estimates of the combinatorial coefficients produced by the decomposition, for they are analogous to the ones found in (3.6) and (3.1).

If we represent all the contributions which admit a bound

$$
C \gamma^{-N\left(1-2 \varepsilon_{0}\right)}
$$

by the following symbol

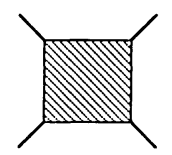

(the number of such contributions is bounded by $C^{n}$, if $n$ is the number of end points of the tree $\gamma$ ), then we have

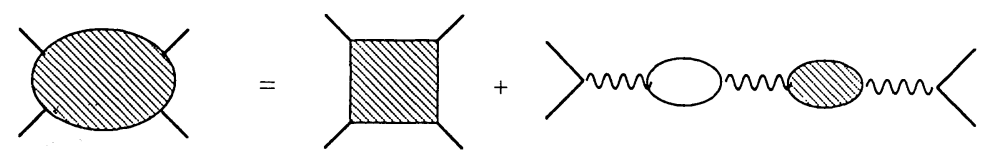

This is tantamount to write, in (2.3), the expression $\mathscr{E}_{(<N)} \mathscr{E}_{(N)}^{T}$ as follows:

$$
\mathscr{E}_{(<N)} \mathscr{E}_{(N)}^{T}=A+B
$$

where $A$ is the sum on a number of contributions $a$ which is bounded by $\leq C^{n}$, and each contribution $a$ admits the bound (2.25) with the substitution

$$
\alpha_{v_{0}} \rightarrow \alpha_{v_{0}}+2\left(1-2 \varepsilon_{0}\right) \delta_{\left|P_{v_{0}}\right|, 4}
$$

$B$ must still be improved. It is shown in Appendix $\mathrm{A} 2$ that, like $A, B$ too admits a factor $\alpha_{v_{0}}^{\prime}$ strictly positive

$$
\alpha_{v_{0}}^{\prime}=\alpha_{v_{0}}+\delta_{\left|P_{v_{0}}\right|, 4} .
$$

The reason for the improvement seats in the symmetry property (1.17) of the covariance. Therefore for the case $\left|P_{v_{0}}\right|=4$ the proof is complete.

Now we proceed analogously for the case $\left|P_{v_{0}}\right|=2$, using the results so far obtained. We have the following decomposition 


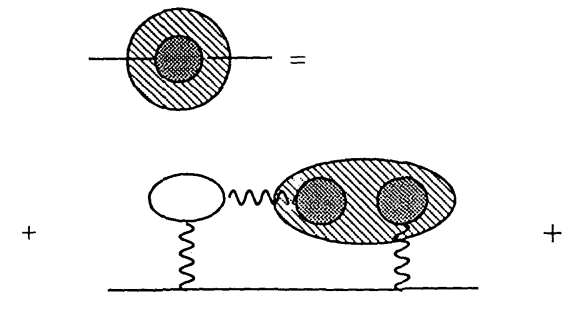

(a)

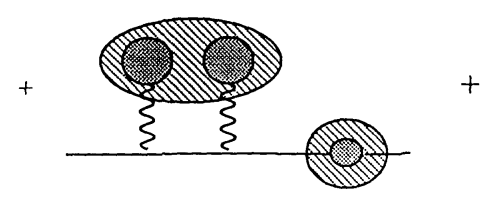

(c)

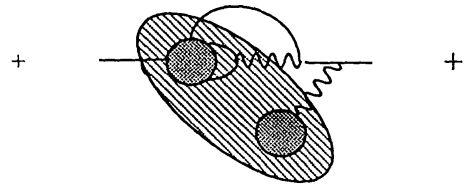

(e)

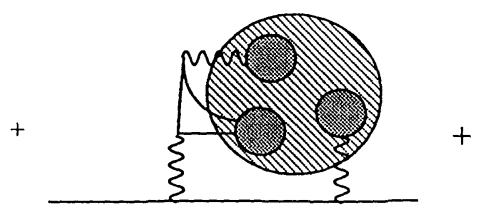

(g)

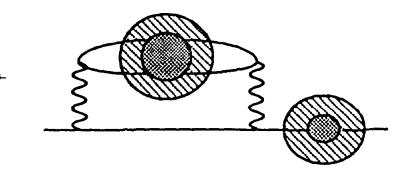

(1)

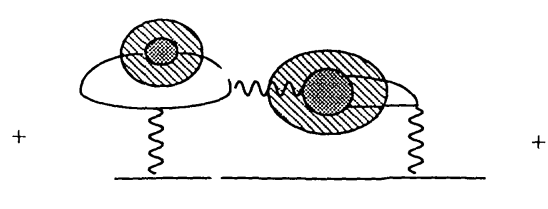

(k)

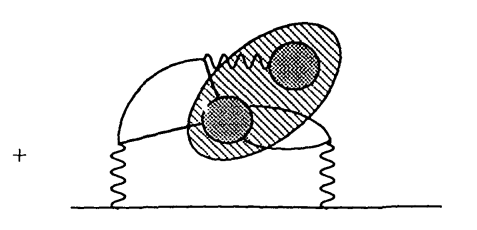

(m)

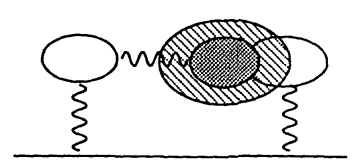

(b)

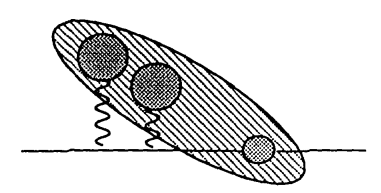

(d)

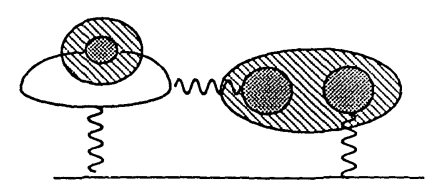

(f)

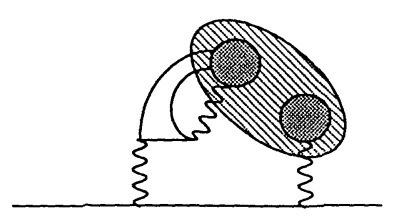

(n)

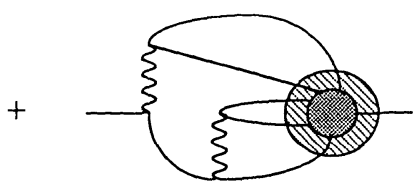

(j)

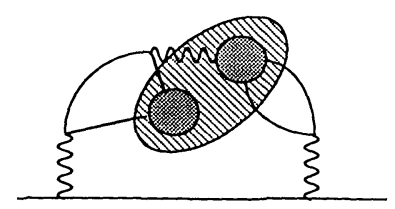

(1)

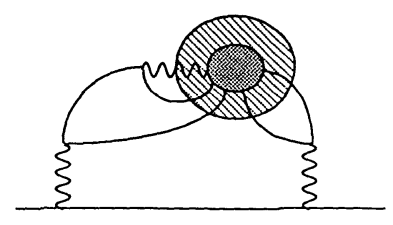

(n) 
and only the first terms (a) and (b) in the above decomposition are not bounded by $C N^{5} \gamma^{-N}$ and need a bound improvement.

Therefore, in analogy to the case $\left|P_{v_{0}}\right|=4$, we can write now

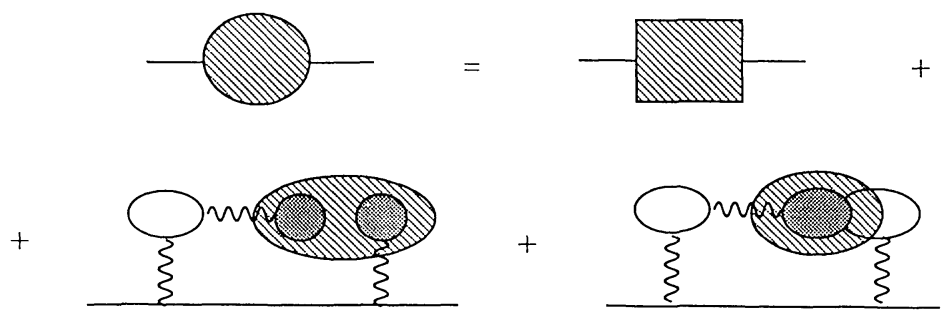

This is tantamount to write

$$
\mathscr{E}_{(<N)} \mathscr{E}_{(N)}^{T}=C+D+E,
$$

where $C$ is the sum on a number of terms $c$ bounded by $\leq C^{n}$, and every term admits the bound (2.25) with the substitution

$$
\alpha_{v_{0}}^{\prime}=\alpha_{v_{0}}+2\left(2-\varepsilon_{2}\right) \delta_{\mid P_{v_{0} \mid, 2}}
$$

where $\varepsilon_{2}=\varepsilon_{0}+\varepsilon_{3}$, if $\varepsilon_{0}$ is given in (3.8) and $\varepsilon_{3}$ is defined by

$$
C N^{5} \gamma^{-N} \leq C \gamma^{-N\left(1-\varepsilon_{3}\right)}
$$

so that we can set $\varepsilon_{3}=5 \varepsilon_{0} \Rightarrow \varepsilon_{2}=6 \varepsilon_{0}$. By reasoning analogous to the case $\left|P_{v_{0}}\right|=4$ and using again crucially the relation (1.17), we show in Appendix A2 how to obtain an improved bound of the terms $D$ and $E$, with the factor $\alpha_{v_{0}}^{\prime}$ given by

$$
\alpha_{v_{0}}^{\prime}=\alpha_{v_{0}}+2\left(1-\varepsilon_{0}\right) \delta_{\mid P_{v_{0} \mid, 2}}+\left(1-2 \varepsilon_{0}\right),
$$

so that the proof is complete also for the case $\left|P_{v_{0}}\right|=2$.

We can now state the following result.

Theorem 3.2. When we define the effective potential on scale $N-1$, if $N$ is the UV cutoff, we find the following bound:

$$
V\left(n, \gamma, P_{v_{0}}\right) \leq \mathscr{C}^{n} \bar{\lambda}^{n} \gamma^{-\frac{1}{2}\left(h_{0}-k\right) \alpha_{v_{0}}^{\prime}} e^{-\frac{\bar{\xi}}{2} d_{(k)}\left(\Delta_{1}, \ldots, \Delta_{\left|P_{v_{0}}\right|}\right)}
$$

where $h_{0}=N$ and $k=N-1 ; \alpha_{v_{0}}^{\prime}$ is given by

$$
\begin{aligned}
& \alpha_{v_{0}}^{\prime}=\alpha_{v_{0}}+\delta_{\left|P_{v_{0}}\right|, 2} \delta_{n_{2, v_{0}, 1}}\left[2\left(1-\varepsilon_{0}\right)\right]+\delta_{\mid P_{v_{0} \mid, 2}} \delta_{n_{2, v_{0}}, 0} \delta_{G_{v_{0}, \mathscr{S}_{2}}}\left[\left(3-4 \varepsilon_{0}\right)\right]
\end{aligned}
$$

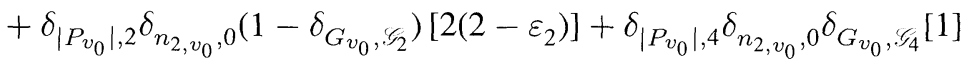

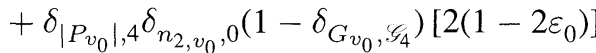

if $\delta_{G_{v_{0}}, \mathscr{G}_{i}}$ is equal to 1 if $G_{v_{0}}=\mathscr{G}_{\imath}$ and is 0 otherwise, with $i=2,4$, and
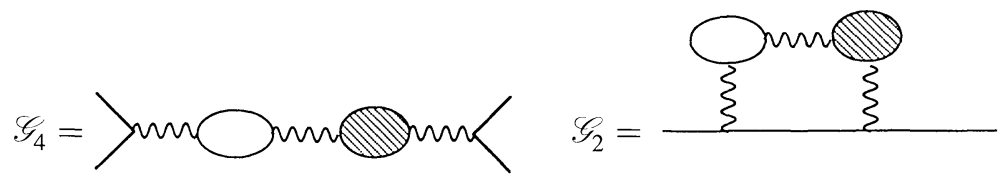
So we can deduce that (2.29) holds with

$$
\eta_{2}=2 \varepsilon_{0}, \quad \eta_{3}=4 \varepsilon_{0}, \quad \eta_{1}=0,
$$

where $\eta_{2}, \eta_{3}$ are sufficiently small, for $\varepsilon_{0}$ sufficiently small. For definiteness we can choose $\eta_{2}, \eta_{3}<1 / 2$.

We would want to outline that, if we consider again trees as in Fig. 3.1, where $k$ is not set equal to $N-1$, but is arbitrary, then we have $h_{0} \in\{k+1, \ldots, N-1, N\}$; nevertheless, in this case too, the bound (3.15) still holds, as it can be argued by decomposing the expectations whose arguments contain any field coming out of graph elements of type $V_{2}$, according to the prescription given in the middle of Sect. 2.

Note that the relation (3.15) has a convergent dependence from $h_{0}-k$ and therefore one can try to obtain similar results in the case of the generic $k<N$ and of the generic tree. This will be the argument of the next section.

\section{Renormalization Group: The General Step}

Now, we consider an arbitrary tree

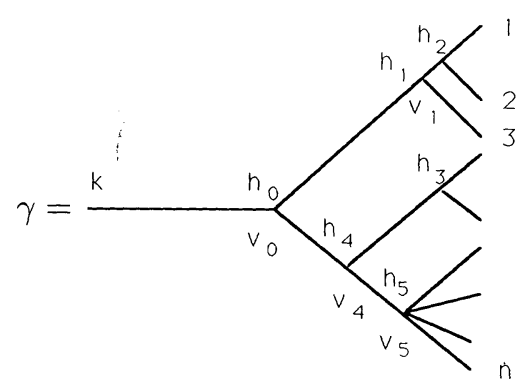

to which there corresponds a class $G_{v_{0}}$ of graphs whose contribution is given by

$$
\prod_{v \geq v_{0}} \frac{\mathscr{E}_{\left(<h_{v}\right)}(\ldots) \mathscr{E}_{\left(h_{v}\right)}^{T}(\ldots)}{s_{v} !}
$$

Here we suppose to have fixed the sets

$$
\left\{\left\{P_{v_{j}}\right\}_{j=1}^{s_{v}},\left\{Q_{v}^{(\jmath)}\right\}_{j=1}^{s_{v}}\right\}_{v \geq v_{0}}
$$

in order to compute (4.1) which, in the end, must be multiplied by a factor

$$
\prod_{j=1}^{n_{4}} \lambda_{j} \prod_{i=n_{4}+1}^{n}\left(\lambda g_{\mathrm{IR}}+\lambda g^{(\leq N)}\right)_{i}
$$

and then integrated. Here and henceforth we don't write explicitly the arguments of the functions and expectation values which we deal with.

We begin by giving some results about the topological structure of the classes of graphs corresponding to (4.2). We have the following fundamental:

Lemma 4.1. Given a vertex $v$ with $\left|P_{v}\right|=2,4$, it is possible to decompose

$$
\mathscr{E}_{\left(<h_{v}\right)}(\ldots) \mathscr{E}_{\left(h_{v}\right)}^{T}(\ldots)=E_{h_{v}}(\ldots)
$$


where $E_{h_{v}}$ is the sum of a number of terms bounded by $C^{s_{v}}$, such that the estimate (2.25) can be replaced with the following one:

$$
\begin{aligned}
V\left(n, \gamma, P_{v_{0}}\right) \leq & \mathscr{E}^{n} \bar{\lambda}^{n} \gamma^{-\frac{k}{2}\left(\alpha_{v_{0}}+\beta_{v_{0}}\right)} e^{-\frac{\bar{\xi}}{2} d_{(k)}\left(\Delta_{1}, \ldots, \Delta_{\left|P_{0}\right|}\right)} \\
& \times \sum_{\left\{h_{v}\right\}} \prod_{v \geq v_{0}}\left\{\sum_{P_{v_{1}} \ldots P_{v_{s_{v}}}} \sum_{Q_{v}^{(1)} \ldots Q_{v}^{(s)}} \gamma^{-\frac{1}{2}\left(\alpha_{v}+\beta_{v}\right)\left(h_{v}-h_{v^{\prime}}\right)}\right\},
\end{aligned}
$$

where $\beta_{v}=2\left[\varrho_{c}+\sigma_{v}\right]$ and

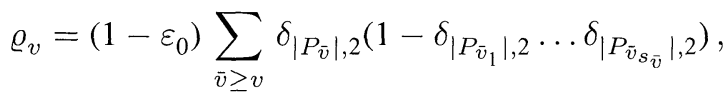

$$
\begin{aligned}
& \sigma_{v}=\sum_{\bar{v} \geq v}\left(\delta_{\left|P_{\bar{v}}\right|, 4}+\delta_{\left|P_{\bar{v}}\right|, 2}\right)\left[1-\prod_{\substack{j=1 \\
P_{\bar{v}_{j}} \cap P_{\bar{v}} \neq \emptyset}}^{s \bar{v}}\left(\delta_{\left|P_{\bar{v}_{j}}\right|, 4}+\delta_{\left|P_{\bar{v}_{j}}\right|, 2}\right)\right] .
\end{aligned}
$$

Note that $\varrho_{v}$ satisfies the inequalities $\varrho_{v} \leq \varrho_{v^{\prime}}$, and $\varrho_{v} \geq\left(1-\varepsilon_{0}\right) \forall v$ such that $\left|P_{v}\right|=2$.

This lemma implies that, for the vertices with $\left|P_{v}\right|=2$, we have an $\alpha_{v}^{\prime} \geq-\varepsilon_{0}$ [compare with (2.28)] and that, when for some $P_{v_{j}}$ such that $P_{v_{j}} \cap P_{v} \neq \emptyset$ we have $\left|P_{v_{j}}\right|>4$, then, for both cases $\left|P_{v}\right|=2$ and $\left|P_{v}\right|=4$, we can write $\alpha_{v}^{\prime}>0$. In both cases the improved bounds are due to the topological structure of the truncated expectations, and not to the symmetry properties of the propagators.

The proof of Lemma 4.1 can be found in Appendix A3. We want to show now, using the results and the ideas of Sect. 3, that actually also for the case $\left|P_{v_{j}}\right| \leq 4 \forall P_{v_{j}}$ such that $P_{v_{j}} \cap P_{v} \neq \emptyset$ it is possible to write an improved bound analogous to (4.4).

The following discussion will be divided into three parts: in the first one we give an algorithm aiming to rewrite the terms in (4.1) corresponding to the vertices of degree zero (see the definition of degree of a vertex in Sect.2) in a more suitable way, just like in Sect. 3. In the second one we state a prescription how to treat the vertices of degree one. Lastly, in the third one we show that in this way the bounds of Sect. 2 can be improved on each scale, so that we can define a factor (2.29) which is strictly positive for each value of $\left|P_{v}\right|$.

Let us start with the vertices $v$ with $d_{1}(v)=0$. For each vertex $v$ there corresponds a cluster $P_{v}$ and a class of graphs $G_{v}$ with $\left|P_{v}\right|$ external legs. The sets (4.2) being fixed, we know which points the external legs of $G_{v_{0}}$ come out of: such points are associated to variables $(t, \vec{x})$ which must be integrated in squares $\Delta_{1}, \ldots, \Delta_{\left|P_{v_{0}}\right|}$ of side $\gamma^{-k}$.

Then, $\forall v, d_{1}(v)=0$, we study the term

$$
\mathscr{E}_{\left(h_{v}\right)}(\ldots) \mathscr{E}_{\left(h_{v}\right)}^{T}(\ldots)
$$

and we decompose it in the following way:

i) if $\left|P_{v}\right| \geq 6 \Rightarrow \mathscr{E}_{\left(h_{v}\right)}(\ldots) \mathscr{E}_{\left(h_{v}\right)}^{T}(\ldots)$ remains unchanged;

ii) if $\left|P_{v}\right|=4 \Leftrightarrow$ we have the graphic representation

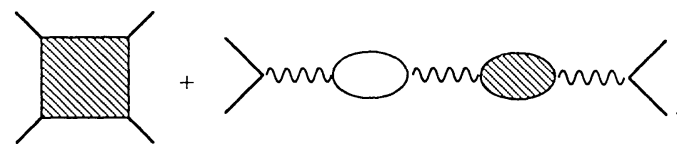


where the contribution (b) is called $\mathscr{G}_{4}$, and is meant as

if $s_{v}=2$;

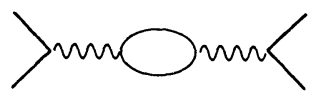

iii) if $\left|P_{v}\right|=2 \Rightarrow$ we have the graphic representation

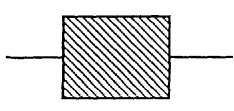

(a)

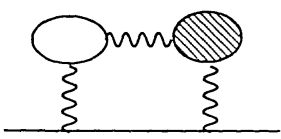

(b)

where the contribution (b) is called $\mathscr{G}_{2}$, and is meant as

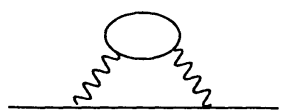

if $s_{v}=2$.

In (4.8), (4.9) the decomposition starts from the point which is chosen according to the following prescription:

i) as far as (4.9) is concerned, only a point can be found in a square $\Delta$; in such a case we choose the other point; if no point is contained in a square, we choose the one which the leg contracted on the lower frequency comes out of;

ii) as far as (4.8) is concerned, we take the point (not contained in a square $\Delta$ ) which the leg contracted on the lowest scale comes out of.

Therefore we have to study (4.1) where the factors corresponding to the vertices with $\left|P_{v}\right|=2,4$ are rewritten according to the decomposition (4.3), the classes of graphs corresponding to vertices $v$ such that $=\left|P_{v}\right| \leq 4$ and $d_{1}(v)=0$ are decomposed like in (4.8), (4.9), and the end points of type $V_{2}$ are treated as it is explained in Sect. 2.

Now we pass to the second part of the section; we consider the vertices $v$ such that $d_{1}(v)=1$. For every vertex $v$ we have a cluster $P_{v}$, and a sequence of clusters $P_{v_{1}}, \ldots, P_{v_{s}}$ contained in $P_{v}$ which must be connected to each other via hard lines $g^{\left(h_{v}\right)}$ and soft lines $g^{\left(<h_{v}\right)}$

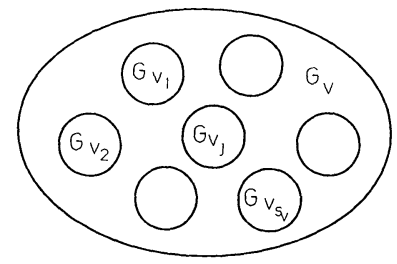

If $\left|P_{v_{j}}\right| \geq 6$ for some $j \in\left\{1, \ldots, s_{v}\right\}$, such that $P_{v_{j}} \cap P_{v} \neq \emptyset$ and $\left|P_{v}\right|=2,4$, we have the decomposition of Lemma 4.1 which guanrantees a convergence factor $\gamma^{-h_{v}}$.

If $\left|P_{v_{j}}\right|=2,4$ for each $j \in\left\{1, \ldots, s_{v}\right\}$, such that $P_{v_{\jmath}} \cap P_{v} \neq \emptyset$, then we distinguish between the following cases.

1) If for some $j \in\left\{1, \ldots, s_{v}\right\}$, such that $P_{v_{j}} \cap P_{v} \neq \emptyset$ we have

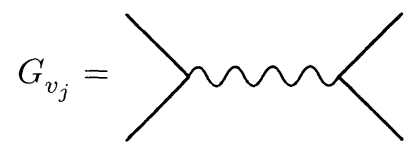


then we choose the point contained in the set

$$
\bigcup_{\substack{j=1 \\ s_{v_{j}}=1}}^{s_{v}} P_{v_{j}} \cap P_{v}
$$

which the external leg contracted on the lowest scale comes out of, and we decompose

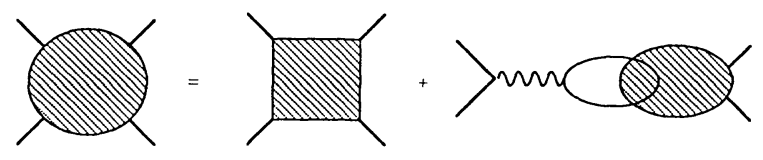

(a)

(b)

where (a) admits a superficial bound with an extra factor $\gamma^{-h_{v}}$, and (b) has a loop $g^{2}$ on scale $h_{v}$.

2) If $\forall v_{\jmath}$ such that $P_{v_{\jmath}} \cap P_{v} \neq \emptyset$, we have

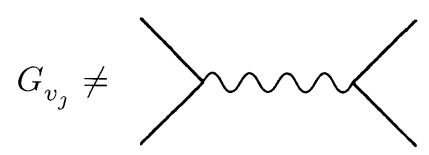

we study all the terms (4.8), (4.9) of the form (b). If $\left|P_{v_{\jmath}}\right|=4, G_{v_{\jmath}}$ is of the form

$$
G_{v_{j}}=\sum_{1}^{x} u^{x} \cos ^{x} u_{2}
$$

where 1 is the point which the external leg contracted on the lowest scale comes out of. The case $1 \in \Delta_{1}, 2 \in \Delta_{2}$ will be discussed later.

Let us first suppose that the two legs coming out of $x_{1}$ are hard lines contracted on the same scale $h_{V}$.

We write

$$
\psi_{t_{1}, \vec{x}_{1}}^{\sigma_{1}}=\psi_{t_{2}, \vec{x}_{1}}^{\sigma_{1}}+\int_{0}^{1} d \alpha\left[\left.\partial_{t} \psi_{t, \vec{x}_{1}}^{\sigma_{1}}\right|_{t=t_{2}+\alpha t_{12}}\right] t_{12}
$$

where $t_{i j} \equiv t_{i}-t_{j}$, and $\sigma_{1}= \pm$, so that we have, for the truncated expectation on scale $h_{V}$,

$$
\begin{aligned}
& \mathscr{E}_{\left(h_{V}\right)}^{T}\left(: \tilde{\psi}^{\left(h_{V}\right)}\left(P_{V_{1}} \backslash Q_{V}^{(1)}\right):, \ldots,: \tilde{\psi}^{\left(h_{V}\right)}\left(P_{V_{s_{V}}} \backslash Q_{V}^{\left(s_{V}\right)}\right):\right) \\
& =\mathscr{E}_{\left(h_{V}\right)}^{T}\left(: \tilde{\psi}^{\left(h_{V}\right)}\left(\left[P_{V_{1}} \backslash Q_{V}^{(1)} \backslash\left(t_{1}, \vec{x}_{1}\right)\right] \cup\left(t_{2}, \vec{x}_{1}\right): \ldots,: \tilde{\psi}^{\left(h_{V}\right)}\left(P_{V_{s_{V}}} \backslash Q_{V}^{\left(s_{V}\right)}\right):\right)\right. \\
& \quad+\int_{0}^{1} d \alpha\left[\partial _ { t } \mathscr { E } _ { ( h _ { V } ) } ^ { T } \left(: \tilde{\psi}^{\left(h_{V}\right)}\left(\left[P_{V_{1}} \backslash Q_{V}^{(1)} \backslash\left(t_{1}, \vec{x}_{1}\right)\right] \cup\left(t, \vec{x}_{1}\right)\right):\right.\right. \\
& \left.\left.\quad \ldots,: \tilde{\psi}^{\left(h_{V}\right)}\left(P_{V_{s_{V}}} \backslash Q_{V}^{\left(s_{V}\right)}\right):\right)\left.\right|_{t+t_{2}+\alpha t_{12}} t_{12}\right]
\end{aligned}
$$

and an analogous expression can be written for the other field $\psi$, so that, in the end, we have (in a symbolic form)

$$
\mathscr{E}_{\left(h_{V}\right)_{0}}^{T}+\delta \mathscr{E}_{\left(h_{V}\right)_{1}}^{T}+\delta \mathscr{E}_{\left(h_{V}\right)_{2}}^{T}+\delta^{2} \mathscr{E}_{\left(h_{V}\right)}^{T}
$$


where there are: i) one term of order zero; ii) two terms of the first order; iii) one term of the second order. They add to the superficial bound of the graphs an extra factor, respectively: i) 1 ; ii) $\gamma^{-\left(h_{v_{j}}-h_{V}\right)}$; iii) $\gamma^{-2\left(h_{v_{j}}-h_{V}\right)}$. Moreover one decomposes

$$
\left.v\left(\vec{y}_{1}-\vec{x}_{1}\right)=v\left(\vec{x}_{2}-\vec{x}_{1}\right)+\left.\int_{0}^{1} d \alpha\left[\partial_{\vec{x}} v(\vec{x})\right]\right|_{\vec{x}=\vec{x}_{2}-\vec{x}_{1}+\alpha\left(\vec{y}_{1}-\vec{x}_{2}\right)}\right]\left(\vec{y}_{1}-\vec{x}_{2}\right),
$$

where the second term allows a gain $\gamma^{-h_{v}}$ and the first one is such that the contribution with $\mathscr{E}_{\left(h_{V}\right)_{0}}^{T}$ in (4.15) gives zero (we have a loop integrated over all $\left.R^{2}\right)$.

If we take in account the exponential decay too, in (4.15) the first order terms, up to a factor $t_{12}$, admit the same bound of $\mathscr{E}_{\left(h_{v}\right)}(\ldots)$ with the following fundamental differences: they have an extra factor $\eta^{h_{V}}$ and a distance (2.12), where $P_{1}$ contains the point $\left(t_{2}+\alpha t_{12}, \vec{x}_{1}\right)$ instead of $\left(t_{1}, \vec{x}_{1}\right)$. Obviously it turns to be

$$
\begin{aligned}
& d_{\mu\left(h_{V}\right)}\left(\left[P_{V_{1}} \backslash\left(t_{1}, \vec{x}_{1}\right)\right] \cup\left(t_{2}+\alpha t_{12}, \vec{x}_{1}\right), P_{V_{2}}, \ldots, P_{V_{s_{V}}}\right) \\
& \quad \geq d_{\mu\left(h_{V}\right)}\left(\left[P_{V_{1}} \backslash\left(t_{1}, \vec{x}_{1}\right)\right] \cup\left(t_{2}, \vec{x}_{1}\right), P_{V_{2}}, \ldots, P_{V_{s_{V}}}\right)-\alpha \gamma^{h} t_{12},
\end{aligned}
$$

where $\alpha \in[0,1]$. In (4.16), the second term, up to a factor $\vec{y}_{1}-\vec{x}_{1}$, admits the same bound of $v$, with the following difference. Instead of

one has

$$
e^{-\left|\vec{x}_{1}-\vec{y}_{1}\right|}
$$

where, obviously

$$
e^{-\left|\vec{x}_{1}-\vec{x}_{2}+\alpha\left(\vec{y}_{1}-\vec{x}_{2}\right)\right|},
$$

$$
\left|\vec{x}_{1}-\vec{x}_{2}+\alpha\left(\vec{y}_{1}-\vec{x}_{2}\right)\right| \geq\left|\vec{x}_{1}-\vec{x}_{2}\right|-\alpha\left|\vec{y}_{1}-\vec{x}_{2}\right| .
$$

The factor $t_{12}$ in (4.13) can be used now to improve by $\gamma^{-h_{v_{j}}}$ the contribution to the bound arising from the integration on that variable. In fact we have an extra exponential whose argument is $\alpha \gamma^{h}\left|t_{12}\right|$, so that we can obtain both the improvement of the dimensional bound and the exponential decay (say with a coefficient $\xi_{0} / 2$ instead of $\bar{\xi} / 2$ where $\xi_{0}<\bar{\xi}$ ).

If one or both legs coming out of $x_{1}$ are soft, but they are contracted on the same scale, one has a similar bound, where one writes the simple expectation too via the interpolation formula.

On the contrary, if the lines coming out of $x_{1}$ are contracted on different scales we repeat the above construction, but we need some more decomposition of the simple and truncated expectations. The situation is the following one

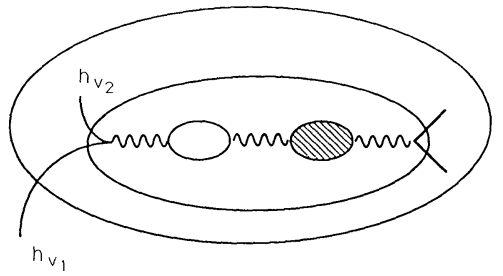

where $h_{V_{1}}<h_{V_{2}} \leq h_{v}$. In every possible case, it is possible to decompose

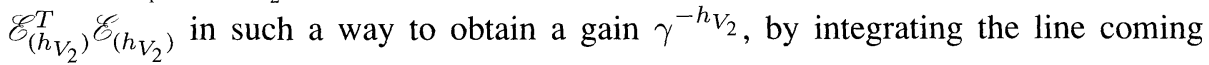


out of $x_{1}$ which is contracted on scale $h_{V_{2}}$, instead of a potential line (this will be always possible, since the two lines coming out of $x_{1}$ cannot form a loop, being contracted on different scales). Therefore we have yet an expression like (4.15), where

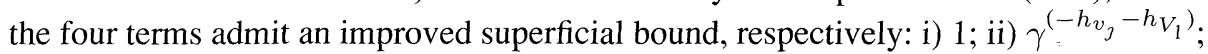
iii) $\gamma^{-\left(h_{v_{j}}-h_{V_{2}}\right)}$; iv) $\gamma^{-\left(h_{v_{\jmath}}-h_{V_{1}}\right)} \gamma^{-\left(h_{v_{\jmath}}-h_{V_{2}}\right)}$, all multiplied by a factor $\gamma^{-h_{V_{2}}}$.

We are left with the case in which the two points 1,2 in (4.12) are in squares $\Delta_{1}$, $\Delta_{2}$. In such a case $G_{v}$ is like in (4.12), with $x_{1} \in \Delta_{1}, 2 \in \Delta_{2}$, where there is at least one point among 1 and 2 (we call it 1), which an external leg of $G_{v}$ comes out of towards some other subcluster inside $G_{v_{0}}$. If there are two such lines, we choose as 1 the point which the leg contracted on the lower scale comes out of. Then let us decompose the field associated to 1 as follows:

$$
\psi_{t_{1}, \vec{x}_{1}}^{\sigma_{1}}=\psi_{t_{2}, \vec{x}_{1}}^{\sigma_{1}}+\int_{0}^{1} d \alpha\left[\left.\partial_{t} \psi_{t, \vec{x}_{1}}^{\sigma_{1}}\right|_{t=t_{2}+\alpha t_{12}}\right] t_{12},
$$

and let us treat the above two contributions separately. As to the first one it is obvious that it is possible to obtain the same bound of Appendix A3,

$$
\gamma^{-\frac{1}{2}\left(h_{v_{j}}-k\right)} e^{-\frac{1}{2} \bar{\xi} d_{(k)}\left(\Delta_{1}, \Delta_{2}\right)}
$$

where we can write

$$
\gamma^{-\frac{1}{2}\left(h_{v_{j}}-k\right)}=\prod_{V \leq v_{j}} \gamma^{-\frac{1}{2}\left(h_{V}-h_{V^{\prime}}\right)}
$$

which guarantee a gain on each scale. As to the second one, the superficial bound gives the convergence factor $\gamma^{-h_{v}} \gamma^{h_{V}}$, where $h_{V}$ is the frequency at which the leg we have chosen is contracted.

If $\left|P_{v}\right|=2$ and $G_{v_{\jmath}}$ is of the form

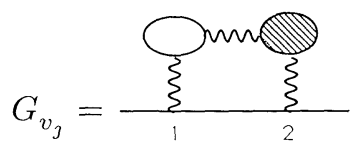

where 1 is the point which the external leg contracted on the lowest scale comes out of, we distinguish between the cases: i) $g_{\lambda}$ hard, and ii) $g_{\lambda}$ soft. If, in (4.21), $g_{\lambda}$ is hard, we write

$$
\begin{aligned}
\psi_{t_{1}, \vec{x}_{1}}^{\sigma_{1}}= & \psi_{t_{2}, \vec{x}_{2}}^{\sigma_{1}}+\int_{0}^{1} d \alpha\left[\left[\left.\partial_{t} \psi_{t, \vec{x}}^{\sigma_{1}}\right|_{\substack{t=t_{2}+\alpha \vec{x}_{2} \\
\vec{x}=t_{12}}}\right] t_{12}\right. \\
& \left.+\left[\left.\partial_{\vec{x}} \psi_{t, \vec{x}}^{\sigma_{1}}\right|_{\substack{t=t_{2} \\
\vec{x}=\vec{x}_{2}+\alpha\left(\vec{x}_{1}-\vec{x}_{2}\right)}}\right]\left(\vec{x}_{1}-\vec{x}_{2}\right)\right]
\end{aligned}
$$

and rewrite the simple or truncated expectation in whose argument the field (4.22) appears as we have done in the case $\left|P_{v}\right|=4$, and obtain two expressions admitting, up to the factors $t_{12}$ and $\left(\vec{x}_{1}-\vec{x}_{2}\right)$, the same bound we had before, multiplied by a factor $\gamma^{h_{V}}$ (which will be won by a convergence factor $\gamma^{-h_{v_{j}}}$ ) and with a distance 
modified as it happened in the above case. We use the fact that (4.22) corresponds to the graph decomposition

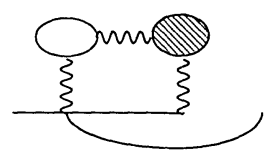

(a)

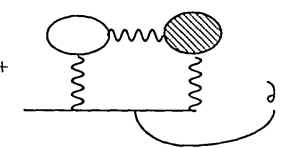

(b)

where (a), integrated, vanishes, while (b) admits a bound improved by a factor $\gamma^{-\left(h_{v_{j}}-h_{V}\right)}$. If, in (4.21), the line $g_{\lambda}$ is soft, we have the following decomposition

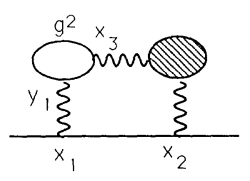

where at least one line in the loop $g^{2}$ is hard, for the graph must be connected. If 1 is the point which the external leg of $G_{v}$ contracted on the lowest scale comes out of, we write

$$
g_{\lambda}\left(t_{12}, \vec{x}_{1}-\vec{x}_{2}\right)=g_{\lambda}\left(t_{32}, \vec{x}_{1}-\vec{x}_{2}\right)+\int_{0}^{1} d \alpha\left[\left.\partial_{t} g_{\lambda}\left(t, \vec{x}_{1}-\vec{x}_{2}\right)\right|_{t=t_{32}+\alpha t_{13}}\right] t_{13}
$$

and

$$
v\left(\vec{x}_{1}-\vec{y}_{1}\right)=v\left(\vec{x}_{1}-\vec{x}_{3}\right)+\int_{0}^{1} d \alpha\left[\left.\partial_{\vec{x}} v(\vec{x})\right|_{\vec{x}=\vec{x}_{1}-\vec{x}_{3}+\alpha\left(\vec{x}_{3}-\vec{y}_{1}\right)}\right]\left(\vec{x}_{3}-\vec{y}_{1}\right)
$$

so that the loop $g^{2}$ becomes free, provided that

$$
\psi_{t_{1}, \vec{x}_{1}}^{\sigma_{1}}=\psi_{t_{3}, \vec{x}_{1}}^{\sigma_{1}}+\int_{0}^{1} d \alpha\left[\left.\partial_{t} \psi_{t, \vec{x}_{1}}^{\sigma_{1}}\right|_{t=t_{1}+\alpha t_{13}}\right] t_{13}
$$

which gives therefore either vanishing contributions or contributions with a gain at least given by

$$
\gamma^{-\left(h_{v_{j}}-h_{V}\right)}
$$

Now we can begin the third part of the section, by considering the vertices of degree $d_{1}(v)>1$. $\forall$ vertex $v$ we have a situation like the following one

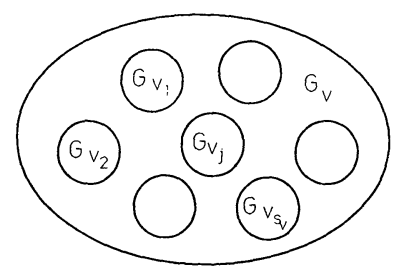


where the classes of graphs $G_{v_{1}}, \ldots, G_{v_{s_{v}}}$ correspond to vertices of degree $d_{1}\left(v_{j}\right)=$ $d_{1}(v)-1$, where $j=1, \ldots, s_{v}$.

In the second part of this section we have shown that, for every cluster corresponding to vertices of degree zero, it is possible, each time we have a number of external legs less or equal to four, to gain a convergence factor $\gamma^{-h_{v}}$ or $\gamma^{-\left(h_{v}-h_{V}\right)}$, where $V$ is a vertex to which there corresponds a cluster $P_{V}$ containing the cluster $P_{v}$. Otherwise, each time we have a cluster corresponding to any vertex of degree zero which more than four external legs come out of, we can arrange the structure of the graphs so that it is still possible to obtain a suitable convergence factor. Since the connection is realized on every scale, we have at least a gain equal to the scale difference $\gamma^{h_{v}-h_{v^{\prime}}}$ for each scale until we reach the first non-trivial vertex $v_{0}$.

We can easily see this property if we reason as follows. Let us consider an arbitrary vertex $v>v_{0}$ and let us suppose $\left|P_{v}\right|=2,4$ so that we need a bound improvement; either there is a vertex $\bar{v} \geq v$ such that, for some $j=1, \ldots, s_{\bar{v}}$ satisfying $P_{\bar{v}_{j}} \cap P_{\bar{v}} \neq \emptyset$, it is $\left|P_{\bar{v}_{j}}\right| \geq 6$, so that we obtain a convergence factor $\gamma^{-h_{\bar{v}}}$; or we can move along the tree until we reach the vertices of degree zero. Then we consider all the clusters $\left\{\tilde{v}: P_{\tilde{v}} \cap P_{v} \neq \emptyset\right\}$, which must have two or four external legs (otherwise we find again the previous case); if $G_{\tilde{v}}$ is of the form (a) of (4.8), (4.9), we obtain a factor $\gamma^{-h_{\tilde{v}}}$; otherwise, if $G_{\tilde{v}}$ is of the form (b) we gain at worst $\gamma^{-\left(h_{\tilde{v}}-h_{V}\right)}$, where $V$ is a vertex whose frequency is lower than $h_{\tilde{v}}$. But, in order to assure the connection of the graph $G_{v_{0}}$ on each scale, at least one of the legs coming out of the collection of graphs corresponding to the vertices $\{\tilde{v}\}$, must be contracted on a scale $h<h_{v}$, so that there exists a vertex $V$ corresponding to such a frequency, i.e. $h_{V}=h$. Therefore we can conclude that in the worst possible case we have at least a gain $\gamma^{-\left(h_{v}-h_{v^{\prime}}\right)}$.

Nevertheless, in the above construction, it can happen that we gain only on the scale differences, so that, since we use only lines on scale $h \geq h_{0}$, in the end we have obtained no gain on the scale difference $h_{0}-k$. In order to avoid such a problem, if $\left|P_{v_{0}}\right|=4$ or $\left|P_{v_{0}}\right|=2$, we give a final decomposition rule, to be applied if we have not obtained any gain on the scale $h_{0}$.

1) If $\left|P_{v_{0}}\right|=4 \Rightarrow$ we write

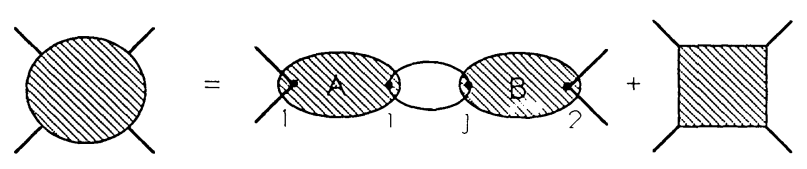

(a)

(b)

where (b) indicates a sum of contributions admitting a good superficial bound, while (a) can be written as follows (under the hypothesis we have obtained no gain on the scale $h_{0}$ the above case is the only possible one requiring a bound improvement)

$$
\begin{aligned}
& \int_{\Delta_{1}} d \vec{x}_{1} d t_{1} \mid \int_{R \times R} d \vec{x}_{i} d t_{i} \int_{R \times R} d \vec{x}_{j} d t_{j} \int_{\Delta_{2}} d \vec{x}_{2} d t_{2} \\
& \quad \times A(1 i) g_{h_{0}}^{2}(i j) B(j 2) \mid,
\end{aligned}
$$


where the meaning of the symbols is obvious. We can rewrite (4.30)

$$
\begin{aligned}
\int_{\Delta_{1}} d & \vec{x}_{1} d t_{1} \mid \int_{R \times R} d \vec{x}_{i 1} d t_{i 1} \int_{R \times R} d \vec{x}_{i j} d t_{i j} \int_{\Delta_{2}-j} d \vec{x}_{2 j} d t_{2 j} \\
& \times A(1 i) g_{h_{0}}^{2}(i j) B(j 2) \mid \\
= & \int_{\Delta_{1}} d \vec{x}_{1} d t_{1} \mid \int_{R \times R} d \vec{x}_{1 i} d t_{i 1} \int_{R \times R} d \vec{x}_{2 j} d t_{2 j} \\
& \times A(1 i) B(j 2) \int_{\Delta_{2}-2 j-i 1-1} d \vec{x}_{i j} d t_{j} g_{h_{0}}^{2}(i j) \mid .
\end{aligned}
$$

Then decompose $A \rightarrow A^{\prime}$ and $B \rightarrow B^{\prime}$ as it must be done for each cluster with four external legs following the rules stated in the first and second parts of this section (now the points $i$ and $j$ are not in squares, but we can estimate the contributions $A$ and $B$ as they would be in). So we compute

$$
\begin{aligned}
& \left|\int_{\Delta_{2}-2 \jmath-\imath 1-1} d \vec{x} d t g_{h_{0}}^{2}(t, \vec{x})\right| \\
& \leq \gamma^{-\frac{1}{2}\left(h_{0}-k\right)} e^{-\frac{1}{2} d_{(k)}\left(1, \Delta_{2}\right)} e^{\frac{1}{2} \gamma^{k}\left(\left|t_{2 j}\right|+\left|t_{i 1}\right|\right)} e^{\frac{1}{2}\left(\left|\vec{x}_{2 j}\right|+\left|\vec{x}_{i 1}\right|\right)} \equiv C^{\prime},
\end{aligned}
$$

and then integrate

$$
\int_{\Delta_{1}} d \vec{x}_{1} d t_{1} \int_{R \times R} d \vec{x}_{i 1} d t_{i 1} \int_{R \times R} d \vec{x}_{2 \jmath} d t_{2 j}\left|A^{\prime}(1 i)\right|\left|C^{\prime}\right|\left|B^{\prime}(j 2)\right|,
$$

where the terms $A^{\prime}$ and $B^{\prime}$ give convergence factors until the scale $h_{0}$, and the term $C^{\prime}$ contains a convergence factor on the scale $h_{0}$.

2) If $\left|P_{v_{0}}\right|=2 \Rightarrow$ we write

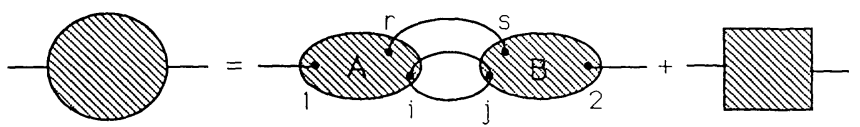

(a)

(b)

where (b) indicates a sum of contributions admitting a good superficial bound, while (a) can be written as follows (under the hypothesis we have obtained no gain on the scale $h_{0}$ the above case is the only possible one requiring a bound improvement)

$$
\begin{aligned}
& \int_{\Delta_{1}} d \vec{x}_{1} d t_{1} \mid \int_{R \times R} d \vec{x}_{i} d t_{i} \int_{R \times R} d \vec{x}_{r} d t_{r} \int_{R \times R} d \vec{x}_{j} d t_{j} \int_{R \times R} d \vec{x}_{s} d t_{s} \\
& \quad \times \int_{\Delta_{2}} d \vec{x}_{2} d t_{2} A(i 1, r 1) g^{2}(j i) g(s r) B(2 j, 2 s) \mid
\end{aligned}
$$

where at least one among the three lines $g$ must be hard, in order to guarantee the graph connection. 
We can study the contribution (b) in (4.33) by reasoning analogous to the discussion of Appendix A4, and in the end we obtain an expression like (4.32), where we have two terms to treat in the same way as all the other terms (so that we gain a convergence factor on each scale $h>h_{0}$ ) and one term which furnishes the convergence factor on scale $h_{0}$. We don't give the details, for the discussion is very similar to the previous one.

As a consequence of what we have said along this section, we can conclude that, in the worst possible case, we have obtained a minimal gain equal to $\gamma^{-\left(h_{v}-h_{v^{\prime}}\right)}$ for each scale $h_{v}>h_{0}$, and a gain equal to $\gamma^{-\frac{1}{2}\left(h_{0}-k\right)}$ for the scale corresponding to the first non-trivial vertex. This gain must to be added to the one we have obtained for the two external leg clusters via the decomposition discussed in the proof of Lemma 4.1. Therefore we can conclude this section by stating the following:

Theorem 4.1. When we define the effective potential on an arbitrary scale $k$, we find the following bound

$$
\begin{aligned}
V\left(n, \gamma, P_{v_{0}}\right) \leq & \mathscr{C}^{n} \bar{\lambda}^{n} \sum_{\left\{h_{v}\right\}} \prod_{v \geq v_{0}}\left\{\sum_{P_{v_{1}} \ldots P_{v_{s}}} \sum_{Q_{v}^{(1)} \ldots Q_{v}^{(s)}}\right. \\
& \left.\times \gamma^{-\frac{1}{2} \alpha_{v}^{\prime}\left(h_{v}-h_{v^{\prime}}\right)} \gamma^{-\frac{k}{2} \alpha_{v_{0}}^{\prime}} e^{-\frac{\xi_{0}}{2} d_{(k)}\left(\Delta_{1}, \ldots, \Delta_{\left|P_{v_{0}}\right|}\right)}\right\},
\end{aligned}
$$

where $\alpha_{v}^{\prime}$ is given by

$$
\alpha_{v}^{\prime}=\alpha_{v}+\beta_{v}+\tau_{v}
$$

if $\alpha_{v}$ is defined as in (2.21), $\beta_{v}$ as in Lemma 4.3, and $\tau_{v}$ has the following properties

$$
\tau_{c}+\sigma_{v} \geq \frac{1}{2}
$$

so that we can deduce (2.29), where $\eta_{1}, \eta_{2}, \eta_{3}$ are sufficiently small. For definiteness we can choose $\eta_{1}, \eta_{2}, \eta_{3}<1 / 2$.

This completes the discussion of the general case, and we end this section by stressing that, since Definition (4.36), Theorem 3.2 can be considered a particular case of Theorem 4.1 for the case $k=N-1$.

Theorem 4.1 proves (2.29) and, therefore, (2.7). In the following section we discuss briefly the modifications we have to bring to the above treatment in order to obtain Theorem 1.1.

\section{Conclusive Remarks}

Until now we have considered the model described by the Hamiltonian $H=H_{0}+V_{0}$, where $V_{0}$ was the sum of the two contributions (1.1), (1.2). We should like to have the possibility of modifying the model by adding a term of the same form of the free Hamiltonian, say

$$
V_{3}=\sum_{\vec{\omega}+ \pm 1} \int d x: \psi_{x, \vec{\omega}}^{+}(-i \alpha \vec{\omega} \vec{\partial}) \psi_{x, \vec{\omega}}^{-}:
$$

and preserving nevertheless the results found in the previous sections. The reason of the above request is explained in [BGM]. In the first part of this section we want to 
show that the renormalization group solution of the ultraviolet problem can be carried exactly as we have done for the model of Sect. 1.

In fact the only difference is that, now, we have also contractions corresponding to hard and soft lines $D^{(n)}$ and $D^{(<n)}$, where

$$
D^{(n)}(x-y, \vec{\omega})=-i \vec{\omega} \vec{\partial} g(x-y, \vec{\omega})
$$

or, analogously

$$
D^{(n)}(k, \vec{\omega})=\vec{\omega} \vec{k} g^{(n)}(k, \vec{\omega}) .
$$

Then it is straightforward to see that the superficial bounds are exactly the same as before, and the only new feature sits in the presence of logarithmically divergent graphs constructed through only vertices of type $\alpha$. If these chains of vertices $\alpha$ are inserted as subgraphs of more complicated graphs it is easy to see that the divergences can be removed using the following properties of the function (5.2).

1) The integral of (5.2) over all $R^{2}$ is vanishing:

$$
\int_{R^{2}} d x D^{(n)}(x, \vec{\omega})=0
$$

because of the integrability of $g^{(n)}$.

2) If we consider a loop $\left(g^{(n)} g^{(m)}\right)(x-y)$ and allow the insertion of an arbitrary number of $\alpha$-vertices, we have a property analogous to (1.17). If we denote by $l(x-y)$ the modified loop, we have

$$
\int_{R^{2}} d x l(x)=0
$$

as is easily obtained in the following way. If $n$ is the total number of contractions forming the loop $l$, and $p=n-2$ is the number of contractions $D$ (the other two lines correspond to contractions $g$ ), we have, trivially

$$
\begin{aligned}
\int_{R^{2}} d x l(x)= & \text { const } \int_{1}^{\gamma^{2}} d \beta_{1} \ldots \int_{1}^{\gamma^{2}} d \beta_{n} \\
& \times \int_{R^{2}} d k e^{-c_{1}\left(\beta_{1}+\ldots+\beta_{n}\right) k^{2}}\left(i k_{0}+\vec{\omega} \vec{k}\right)^{n}(\vec{\omega} \vec{k})^{p}
\end{aligned}
$$

where $c_{1}$ is a constant. If we recall the formula for the Hermite polynomials $H_{n}$ :

$$
H_{n}(\vec{\omega} \vec{k})=\frac{1}{\sqrt{\pi}} \int_{-\infty}^{\infty} d k_{0} e^{-k_{0}^{2}}\left(i k_{0}+\vec{\omega} \vec{k}\right)^{n}
$$

where the polynomial normalization is that the integral of $H_{n}^{2}(x)$ with weight $\exp \left(-x^{2}\right)$ is $2^{-n} n$ ! (Wick rule), then we have immediately, for the orthonormality of the Hermite polynomials, that (5.6) is identically vanishing, which is property we wanted.

3) Eventually, the covariance (5.3) is exponentially decreasing at infinity and is bounded everywhere:

$$
\left|D^{(n)}(x, \vec{\omega})\right| \leq \gamma^{2 n} G_{0} e^{-\gamma^{n}|x|}
$$


where the extra factor $\gamma^{n}$ appearing with respect to the first equation of (1.18) is responsable for the fact that the superficial bounds are neither improved nor destroyed by the new terms corresponding to the $\alpha$-vertices.

Therefore the only graphs we have to consider are the graphs with only $\alpha$ vertices. This class of graphs introduces effectively an ultraviolet divergence in the effective potential, but this is expected, and induces a modification in the two point Schwinger functions that consists simply in a different constant in front of it. See also [BGPS] for an identical discussion.

Finally let us discuss the modifications to the proof of the convergence of the effective potential due to the presence of the propagators $g_{N}^{(n)}(n>N)$ and $\bar{g}_{N}^{(n)}(n<N)$ of Sect. 1.

This is easily done because of the two following remarks:

1) If one computes the contribution of a graph with one or more lines $g^{(n)}$ substituted with lines $g^{(n)_{N}}$ or $\bar{g}_{N}^{(n)}$, the improvement to the superficial bound due to the oddness of the propagator, represented by the factor (4.5) in Lemma 4.1, still holds. This implies that only logarithmic divergences may appear in the contributions given by such graphs.

2) The logarithmic divergences are in fact absent, because of the scaling properties of $g_{N}^{(n)}$ and $\bar{g}_{N}^{(n)}$. The first in fact scales as $\gamma^{N}$ independently from $n$, and decays on scale $n$ in the time direction, while the latter has the extra factor $\gamma^{-2(N-n)}$. In both cases if one performs the sum on the frequencies of the modified lines (remember that for $g_{N}^{(n)}$ the sum is from $N$ up to infinity, while for $\bar{g}_{N}^{(n)}$ is from 0 up to $N$ ) one immediately sees that the contribution of any graph is in fact finite, and no further improvements are needed in these cases. This concludes our discussion.

\section{Appendix A1}

We want to prove, if we define

$$
S\left(P_{v_{0}}, \gamma, n\right)=\prod_{v \geq v_{0}} \sum_{\left\{P_{v_{j}}\right\}_{j=1}^{s_{v}}} \sum_{\left\{Q_{v}^{(j)}\right\}_{j=1}^{s_{v}}} \gamma^{-\frac{1}{2}\left|P_{v}\right|}
$$

the following bound

$$
S\left(P_{v_{0}}, \gamma, n\right) \leq C_{4}^{n}
$$

where $C_{4}$ is a constant.

Let us write

$$
\begin{aligned}
& S\left(P_{v_{0}}, \gamma, n\right)=\prod_{v \geq v_{0}} \sum_{p_{v_{1}}, \ldots, p_{v_{s}}} \sum_{q_{v}^{(1)}, \ldots, q_{v}^{(s)}} \gamma^{-\frac{1}{2} p_{v}} \chi\left(\text { constraint on }\left\{p_{v_{\jmath}}\right\},\left\{q_{v}^{(j)}\right\}\right) \\
& \times \prod_{v \geq v_{0}} \sum_{\left\{P_{v_{j}}\right\}_{j=1}^{s v}} \sum_{\left\{Q_{v}^{(j)}\right\}_{j=1}^{s v}} 1 \\
& \left|P_{v_{j}}\right|=p_{v_{j}}\left|Q_{v}^{(\jmath)}\right|=q_{v}^{(\jmath)}
\end{aligned}
$$

where $\chi$ reveals the presence of the following constraint:

$$
q_{v}^{(j)} \in\left[0, p_{v_{\jmath}}-1\right], \quad p_{v_{j}} \in\left[1, \sum_{j^{\prime}=1}^{s_{v_{j}}} q_{v}^{\left(j^{\prime}\right)}\right] .
$$


We obtain easily an upper bound if: i) we ignore the presence of $\chi$; ii) we remove the constraint on $P_{v_{0}}$, i.e. we sum on all the $P_{v_{0}}$. So, rearranging the sums and bearing in mind that we are summing from the vertex $v_{0}$ towards the end points, we will have

$$
\begin{aligned}
S\left(P_{v_{0}}, \gamma, n\right) \leq & \prod_{v \geq v_{0}} \sum_{p_{v}} \sum_{q_{v}^{(1)}, \ldots, q_{v}^{\left(s_{v}\right)}} \gamma^{-\frac{1}{2} p_{v}} \\
& \times \sum_{\substack{P_{v}:\left|P_{v}\right|=p_{v} \\
P_{v} \subset \bigcup_{j=1}^{s v} Q_{v}^{(j)}}} \sum_{\substack{\left.Q_{v}^{(\jmath)}\right\}_{j=1}^{s v}:\left|Q_{v}^{(j)}\right|=q_{v}^{(j)} \\
Q_{v}^{(j)} \subset P_{v j}}} 1 \equiv \Sigma(\gamma, n) .
\end{aligned}
$$

The last line in (A1.4) gives

$$
\left(\begin{array}{c}
\sum_{j=1}^{s_{v}} p_{v}^{(j)} \\
p_{v}
\end{array}\right) \prod_{j=1}^{s_{v}}\left(\begin{array}{c}
p_{v_{\jmath}} \\
q_{v}^{(j)}
\end{array}\right)
$$

If we write

$$
\Sigma(\gamma, n)=\prod_{v \geq v_{0}} I_{v}
$$

we can give the term corresponding to the vertex $v_{0}$ the following expression

$$
\begin{aligned}
I_{v_{0}} & =\sum_{\left\{q_{\left.\left.v_{0}\right\}_{\jmath}\right\}_{v_{0}}}\right.} \sum_{p_{v_{0}}} \gamma^{-\frac{p_{v_{0}}}{2}}\left(\begin{array}{c}
\sum_{j=1}^{s_{v_{0}}} p_{v_{0}}^{(j)} \\
p_{v_{0}}
\end{array}\right) \prod_{j=1}^{s_{v_{0}}}\left(\begin{array}{c}
p_{v_{\jmath}} \\
q_{v_{0}}^{(j)}
\end{array}\right) \\
& =\sum_{\left\{q_{\left.\left.v_{0}\right\}_{\jmath}\right\}_{v_{0}}}\right.} \prod_{j=1}^{s_{v_{0}}}\left(\begin{array}{c}
p_{v_{\jmath}} \\
q_{v_{0}}^{(\jmath)}
\end{array}\right)\left[1+\gamma^{-\frac{1}{2}}\right]^{p_{v_{\jmath}}} \\
& =\prod_{j=1}^{s_{v_{0}}}\left[2\left(1+\frac{1}{2 \gamma^{1 / 2}}\right)\right]^{p_{v_{\jmath}}}
\end{aligned}
$$

so that

$$
\Sigma(\gamma, n)=\prod_{j=1}^{s_{v_{0}}}\left[2\left(1+\frac{1}{2 \gamma^{1 / 2}}\right)\right]^{p_{v_{j}}} \prod_{v>v_{0}} I_{v}
$$

Now we proceed in the same way for $I_{v_{j}}, j=1, \ldots, s_{v_{0}}$, where $\left\{v_{j}\right\}_{j=1}^{s_{v_{0}}}$ is the collection of vertices immediately following $v_{0}$ along the tree $\gamma$,

$$
I_{v_{0}} \prod_{j=1}^{s_{v_{0}}} I_{v_{j}}=\prod_{j=1}^{s_{v_{0}}} \prod_{i=1}^{s_{v_{j}}}\left[2+\frac{1}{\gamma^{1 / 2}}\left(2+\frac{1}{\gamma^{1 / 2}}\right)\right]^{p_{v_{\imath \jmath}}}
$$

where $\left\{v_{i j}\right\}_{i=1}^{s_{v_{j}}}$ is the collection of vertices following $v_{j}$; and so on, until, if we denote by $\mathscr{P}$ a path from the root of the tree to an end point (we have $n$ such paths), and 
by $l(\mathscr{P})$ the number of vertices on $\mathscr{P}$, we shall have

$$
\begin{aligned}
\Sigma(\gamma, n) & \leq \prod_{\mathscr{P}}\left[2+\frac{1}{\gamma^{1 / 2}}\left(2+\frac{1}{\gamma^{1 / 2}}\left(2+\frac{1}{\gamma^{1 / 2}}(\ldots)\right)\right)\right]^{4} \\
& =\prod_{\mathscr{P}}\left[2\left(\sum_{k=0}^{l(\mathscr{P})-1} \gamma^{-\frac{k}{2}}+\frac{1}{2} \gamma^{-\frac{l(\mathcal{P})}{2}}\right)\right]^{4} \\
& \leq\left(\frac{2 \gamma^{1 / 2}}{\gamma^{1 / 2}-1}\right)^{4 n}=C_{n}^{4}
\end{aligned}
$$

where, e.g., $C_{4}=(4 \gamma)^{2}\left[1-\gamma^{-\frac{1}{2}}\right]^{-1}$. Therefore (A2.4), (A2.5) give

$$
S\left(P_{v_{0}}, \gamma, n\right) \leq C_{4}^{n}
$$

which proves the bound (2.27). Q.E.D.

\section{Appendix A2}

Let us start with the bound of the contribution

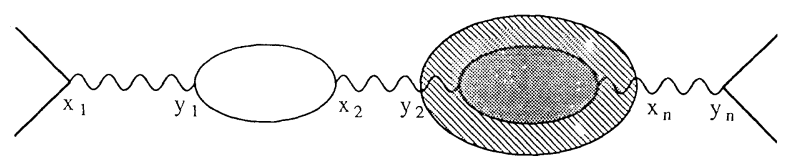

which corresponds to the analytic expression

$$
I=\frac{1}{n !} C^{n} \int_{\substack{x_{1} \in \Delta_{1} \\ y_{n} \in \Delta_{2}}} \prod_{\imath=1}^{n} d x_{\imath} d y_{\imath} \prod_{i=1}^{n} \lambda\left(x_{\imath}-y_{\imath}\right)\left(g g^{(N)}\right)\left(y_{1}-x_{2}\right) \mathscr{A}\left(y_{2}, \ldots, x_{n}\right),
$$

where here and henceforth we can suppose $n_{2}=0$, because we have seen this is the only case in which we must improve the bound, and

$$
\mathscr{t}\left(y_{2}, \ldots, x_{n}\right)=\mathscr{E}_{(<N)}(\ldots) \mathscr{E}_{(N)}^{T}(\ldots)
$$

and $g$ can be $g^{(N)}$ or $g^{(<N)}$. Then we write

$$
A\left(y_{2}-x_{n}\right)=\int \prod_{i=3}^{n-1} d x_{i} d y_{i} \prod_{i=3}^{n-1} \lambda\left(x_{i}-y_{i}\right) . \not b\left(y_{2}, \ldots, x_{n}\right)
$$

so that (A2.2) becomes

$$
\begin{aligned}
I= & \frac{1}{n !} C^{n} \int_{\substack{x_{1} \in \Delta_{1} \\
y_{n} \in \Delta_{2}}} d \vec{x}_{1} d \vec{y}_{1} d t_{1} d \vec{x}_{2} d \vec{y}_{2} d t_{2} d \vec{x}_{n} d \vec{y}_{n} d t_{n} v\left(\vec{x}_{1}-\vec{y}_{1}\right) \\
& \times\left(g g^{(N)}\right)\left(t_{1}-t_{2}, \vec{y}_{1}-\vec{x}_{2}\right) v\left(\vec{x}_{2}-\vec{y}_{2}\right) A\left(t_{2}-t_{n}, \vec{y}_{2}-\vec{x}_{n}\right) v\left(\vec{x}_{n}-\vec{y}_{n}\right) .
\end{aligned}
$$

We have named $\Delta_{1}, \Delta_{2}$ the two squares of side size $\gamma^{-k}$, with $k=N-1$, in which the points $x_{1}, y_{n}$, respectively, vary in (A2.2). In order to bound (A3.3) we distinguish between the following three cases. 
1) Time distance between $\Delta_{1}, \Delta_{2}$ larger than $\gamma^{-k}$ : in this case we can use the superficial bounds as always, so obtaining an expression which contains a factor

$$
e^{-\frac{1}{2} \bar{\xi} d_{(k)}^{1}\left(\Delta_{1}, \Delta_{2}\right)} e^{-\frac{3}{4} \gamma^{N} \bar{\xi} d_{(k)}^{0}\left(\Delta_{1}, \Delta_{2}\right)},
$$

where we have rewritten (2.8) as

$$
d_{(k)}^{3}\left(P_{v_{0}}\right)=\sum_{i=1}^{\left|P_{v_{0}}\right|-1}\left|\lambda_{j}^{(i)}\right|, \quad j=0,1,
$$

and have used the fact that, obviously, instead of $\exp (A)=\exp (A / 2) \exp (A / 2)$, we can write $\exp (A)=\exp (A / 4) \exp (3 A / 4)$, then extract a factor $\exp (3 A / 4)$ and integrate the remaining expression. It will yield simply an extra constant $C^{n}$, where $C=2$.

So we have

$$
e^{-\frac{3}{4} \gamma^{N} \bar{\xi} d_{(k)}^{0}\left(\Delta_{1}, \Delta_{2}\right)} \leq e^{-\frac{1}{2} \gamma^{N} \bar{\xi} d_{(k)}^{0}\left(\Delta_{1}, \Delta_{2}\right)} \frac{C}{\gamma^{N} \gamma^{k}},
$$

and we have gained a convergence factor $\gamma^{-(N-k)}$.

2) Squares $\Delta_{1}, \Delta_{2}$ contiguous in time: in this case $d_{(k)}^{0}\left(\Delta_{1}, \Delta_{2}\right)=0$; we construct, in $\Delta_{1}$, a strip adjacent in time to $\Delta_{2}$ whose thickness is given by

$$
r=\varepsilon \gamma^{-k}=\gamma^{-\frac{1}{2}(N-k)} \gamma^{-k}=\gamma^{-\frac{1}{2}(N+k)}
$$

so that

$$
\begin{gathered}
\Delta_{1}=\delta_{1} \cup \delta_{2}, \\
\delta_{1}=\left\{t_{1} \in \Delta_{1}(t):\left|t_{1}-\Delta_{2}(t)\right|>r\right\} \\
\delta_{2}=\Delta_{1} \backslash \delta_{1}
\end{gathered}
$$

where we have set

$$
\Delta_{\imath}=\Delta_{\imath}(\vec{x}) \times \Delta_{\imath}(t), \quad i=1,2
$$

and, if we write

$$
\int_{\Delta_{1}(t)} d t=\int_{\delta_{1}} d t+\int_{\delta_{2}} d t
$$

and we bound one by one the two contributions we have obtained from (A2.2), we find in the end for both of them the following result:

$$
C e^{-\frac{1}{2} \bar{\xi} d_{(k)}^{1}\left(\Delta_{1}, \Delta_{2}\right)} \gamma^{-\frac{1}{2}(N-k)}
$$

3) Squares $\Delta_{1}, \Delta_{2}$ coinciding in time: in this case $\Delta_{1}(t) \equiv \Delta_{2}(t)$ and $d_{(k)}^{0}\left(\Delta_{1}\right.$, $\left.\Delta_{2}\right)=0$. If $t_{n}$ is such that

$$
t_{n} \in \Delta_{1}^{a}(t) \equiv\left\{t_{n} \in \Delta_{1}(t): d\left(t_{n}, R \backslash \Delta_{1}(t)\right)>r\right\},
$$

we define

$$
\delta_{1}=\left[t_{n}-r, t_{n}+r\right], \quad \delta_{2}=\Delta_{1}(t) \backslash \delta_{1},
$$

where $r$ is given by (A2.5). We define moreover

$$
\Delta_{1}^{b}(t)=\Delta_{1}(t) \backslash \Delta_{1}^{a}(t) .
$$


Now we write

$$
\int_{\Delta_{1}(t)} d t_{1} \int_{\Delta_{1}(t)} d t_{n}=\int_{\Delta_{1}^{a}(t)} d t_{n}\left[\int_{\delta_{1}} d t_{1}+\int_{\delta_{2}} d t_{1}\right]+\int_{\Delta_{1}^{b}(t)} d t_{n} \int_{\Delta_{1}(t)} d t_{1}
$$

so that (A2.2) gives three contributions which we bound separately. For all three we obtain in the end the bound

$$
C e^{-\frac{1}{2} \bar{\xi} d_{(k)}^{1}\left(\Delta_{1}, \Delta_{2}\right)} \gamma^{-\frac{1}{2}(N-k)}
$$

As far as the second and the third contributions are concerned, the result is a mere consequence of the two cases discussed above. The treatment of the first, more interesting contribution is conceptually very similar, albeit technically a little more involved. We sketch the proof of (A2.6) for such contribution. One writes $v\left(\vec{x}_{1}-\vec{y}_{1}\right)$ in (A2.4) as sum of two parts

$$
v\left(\vec{x}_{1}-\vec{x}_{2}\right)+\left[v\left(\vec{x}_{1}-\vec{y}_{1}\right)-v\left(\vec{x}_{1}-\vec{x}_{2}\right)\right],
$$

where the second one can be rewritten via the interpolation formula

$$
\left.v(\vec{x})-v(\vec{y})=\int_{0}^{1} d \alpha v^{\prime}(\vec{x}+\alpha(\vec{y}-\vec{x}))(\vec{y}-\vec{x})\right)
$$

(if $v^{\prime}$ denotes the first derivative of $v$ ) and can be shown to improve the superficial bound by a convergence factor $\gamma^{-N}$. The remaining term has the following property: if we integrate on $t_{1}$ over all $R$, instead of $\delta_{1}$, it vanishes $\Rightarrow$ then we can replace the integration over $\delta_{1}$ with the integration over the complementary subset $R \backslash \delta_{1}$, so that it becomes $\left|t_{1}-t_{n}\right|>r$, and the calculation is now performed in the same way as the case $t_{1} \in \delta_{2}$.

Therefore the class of graphs (A2.1) can be bounded by the following expression:

$$
C^{n} \bar{\lambda}^{n} e^{-\frac{1}{2} \bar{\xi} d_{(k)}\left(\Delta_{1}, \Delta_{2}\right)} \gamma^{-\frac{1}{2}(N-k)}
$$

where $k=N-1$.

Now we study the following graphs:

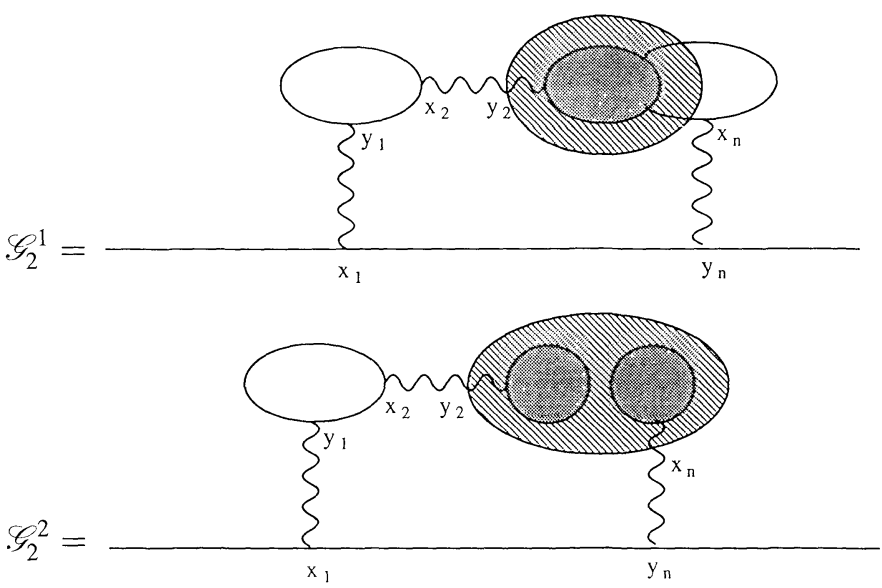


where, in $\mathscr{G}_{2}^{2}$, among $l_{1}, l_{2}, l_{3}$ at least one must be a hard line. As above we write the analytic expressions which correspond to the graphs $\mathscr{I}_{2}^{1}$ and $\mathscr{G}_{2}^{2}$ :

$$
\begin{aligned}
I_{1}= & \frac{1}{n !} C^{n} \int_{\substack{x_{1} \in \Delta_{1} \\
y_{n} \in \Delta_{2}}} \prod_{i=1}^{n} d x_{\imath} d y_{i} \prod_{i=1}^{n} \lambda\left(x_{\imath}-y_{\imath}\right) \\
& \times\left(g g^{(N)}\right)\left(y_{1}-x_{2}\right) g^{(N)}\left(x_{1}-y_{n}\right) \mathscr{b}_{1}\left(y_{2}, \ldots, x_{n}\right), \\
I_{2}= & \frac{1}{n !} C^{n} \int_{\substack{x_{1} \in \Delta_{1} \\
y_{n} \in \Delta_{2}}} \prod_{i=1}^{n} d x_{i} d y_{i} \prod_{i=1}^{n} \lambda\left(x_{i}-y_{i}\right) \\
& \times\left(g g^{(N)}\right)\left(y_{1}-x_{2}\right) g_{l_{1}}\left(x_{1}-y_{n}\right) \mathscr{A}_{2}\left(y_{2}, \ldots, x_{n-1}\right) g_{l_{2}} g_{l_{3}},
\end{aligned}
$$

where the meaning of the symbols is obvious.

It is not difficult to study $I_{1}$ and $I_{2}$ with the same techniques discussed above, but one must be a little more careful when the case $\Delta_{1}=\Delta_{2}$ is considered as far as the term $I_{1}$ is concerned. Let us sketch how to treat this case. We write

$$
\begin{gathered}
\Delta_{1}=\Delta_{1}^{a} \cup \Delta_{1}^{b}, \\
\Delta_{1}^{a}=\left\{x_{1} \in \Delta_{1}: d\left(x_{1}, R^{2} \backslash \Delta_{1}\right)>r\right\}, \Delta_{1}^{b}=\Delta_{1} \backslash \Delta_{1}^{a},
\end{gathered}
$$

and for $x_{1} \in \Delta_{1}^{a}$ :

$$
\begin{gathered}
\delta_{1}=\left\{y_{n} \in \Delta_{2}: d\left(x_{1}, y_{n}\right)<r\right\}, \\
\delta_{2}=\Delta_{2} \backslash \delta_{1} .
\end{gathered}
$$

So we can write $I_{1}$ as the sum of three contributions, because

$$
\int_{\Delta_{1}} d x_{1} \int_{\Delta_{1}} d y_{n}=\int_{\Delta_{1}^{a}} d x_{1}\left[\int_{\delta_{1}} d y_{n}+\int_{\delta_{2}} d y_{n}\right]+\int_{\Delta_{1}^{b}} d x_{1} \int_{\Delta_{1}} d y_{n}
$$

and, as in (A2.6), we obtain for every term the bound

$$
N \gamma^{-\frac{1}{2}(N-k)}
$$

Similar bounds can be obtained for the other terms of $I_{1}$ and for $I_{2}$. So we have found that the class of graphs in (A2.7) and (A2.8) can be bounded by the following expression:

$$
C^{n} \bar{\lambda}^{n} e^{-\frac{1}{2} \bar{\xi} d_{(k)}\left(\Delta_{1}, \Delta_{2}\right)} \gamma^{-\frac{1}{2}(N-k)\left(1-4 \varepsilon_{0}\right)}
$$




\section{Appendix A3}

Consider a vertex $v$, with $\left|P_{v}\right|=2$, to which there corresponds the expression

$$
\begin{gathered}
\mathscr{E}_{\left(<h_{v}\right)}\left(\prod_{j=1}^{s_{v}}: \tilde{\psi}^{\left(<h_{v}\right)}\left(Q_{v}^{(\jmath)} \backslash P_{v} \cap Q_{v}^{(\jmath)}\right):\right) \\
\mathscr{E}_{\left(h_{v}\right)}^{T}\left(: \tilde{\psi}^{\left(h_{v}\right)}\left(P_{v_{1}} \backslash Q_{v}^{(1)}\right):, \ldots,: \tilde{\psi}^{\left(h_{v}\right)}\left(P_{v_{s v}} \backslash Q_{v}^{\left(s_{v}\right)}\right):\right),
\end{gathered}
$$

where $P_{v_{1}}, \ldots, P_{v_{s}}$ are the $s_{v}$ subclusters contained in $P_{v}$, connected to each other on scale $h_{v}$. Let us call 1 the point which one of the two external legs of $P_{v}$ comes out of, and $P_{v_{1}}$ the subcluster which it belongs to:

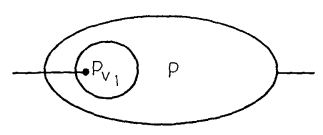

We distinguish in what follows the two possible cases $\left|P_{v_{1}}\right|=2$ and $\left|P_{v_{1}}\right|>2$. 1) Case $\left|P_{v_{1}}\right|>2$ : we divide $P_{v_{1}}$ into two clusters $R_{1}, R_{2}$ :

$$
P_{v_{1}}=R_{1} \cup R_{2}
$$

such that $R_{1}$ contains the point 1 and both $R_{1}$ and $R_{2}$ contain a point which a line (soft or hard) connecting $P_{v_{1}}$ to any other subcluster of $P_{v}$ comes out of; such points exist undoubtedly, for not more than two lines come out of 1 , and we are supposing $\left|P_{v_{1}}\right|>2$, and they are distinct, for otherwise the corresponding graphs vanish for parity reasons. Since the sets (4.3) are fixed, we are making no choice, and therefore no sum.

Now it is easy to see that we can write a decomposition of (A3.1) in the following way:

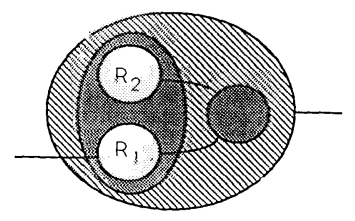

(a)

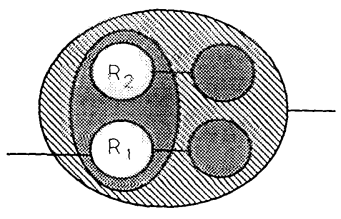

(b)

In (A3.2) one of the two lines drawn in the figure (a) can be soft.

We write symbolically the (A3.2) saying that, if $\left|P_{v}\right|=2$, and $\left|P_{v_{1}}\right|>2$,

$$
\mathscr{E}_{\left(<h_{v}\right)}(\ldots) \mathscr{E}_{\left(h_{v}\right)}^{T}(\ldots)=A(\ldots)
$$

where we have not written explicitly the arguments of the above functions, and $A(\ldots)$ is the sum of a number of terms bounded by $C^{s_{v}}$.

Let us see now why the writing $A(\ldots)$ is more suitable. If we consider (A3.1), fix a variable and integrate over all the other (free) variables we meet moving along the 
anchored tree $\mu$ which connects to each other the subclusters, we obtain

$$
\begin{aligned}
& \int \prod_{j=2}^{s_{v}} d \bar{z}_{j} d t_{j}\left|\mathscr{E}_{\left(<h_{v}\right)}(\ldots) \mathscr{E}_{\left(h_{v}\right)}^{T}(\ldots)\right| \\
& \gamma^{\frac{h_{v}}{2}\left(\sum_{j}\left|P_{v_{j}}\right|-\left|P_{v}\right|\right)} C_{0}^{\left(\sum_{j}\left|P_{v_{j}}\right|-\left|P_{v}\right|\right)} \sum_{\mu} \int \prod_{j=2}^{s_{v}} d \bar{z}_{j} d t_{j} e^{-\xi d_{\mu\left(h_{v}\right)}\left(P_{v_{1}}, \ldots, P_{v_{s_{v}}}\right)} \\
& <s_{v} ! \gamma^{\frac{h_{v}}{2}\left(\sum_{j}\left|P_{v_{j}}\right|-\left|P_{v}\right|\right)} C_{0}^{\left(\sum_{j}\left|P_{v_{j}}\right|-\left|P_{v}\right|\right)} C^{s_{v}-1} \gamma^{-2 h_{v}\left(s_{v}-1\right)} .
\end{aligned}
$$

On the contrary, as far as the term $A(\ldots)$ is concerned, we proceed nearly in the same way, but with the following fundamental difference: the analytic expression $A(\ldots)$ corresponds to the situation (A3.2) where there is a point $Q$ contained in $R_{2}$ or in $R_{1}$ which an external leg of $P_{v_{1}}$ comes out of. Obviously there must be an ondulated line (representing a potential line) either directly connected to such a point or connected to a graph element of type $V_{2}$ which is directly connected to such a point (if there is more than one graph element of type $V_{2}$ there are no convergence problems and no decomposition of the truncated expectation is required). If $\left|P_{v} \cap P_{v_{1}}\right|=1$, then we can delete such a line, for it is inessential for the hard connectedness of the graph, and use the remaining available integration for the line obtained by connecting the external leg to an external leg of any other subcluster of $P_{v}$. Otherwise, if $\left|P_{v} \cap P_{v_{1}}\right|=2$, it can happen that the considered potential line is used to assure the hard connectedness of the subgraph $G_{v_{1}}$; this situation can present itself if there is also an external line of $G_{v_{1}}$ coming out of the other point $Q^{\prime}$ connected to $Q$ through the potential line:

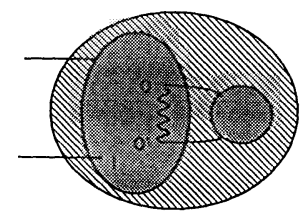

(a)

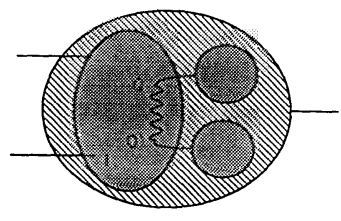

(b)

(obviously we can have more lines external to $G_{v_{1}}$ than the drawn ones). Then we can see that in this case too it is possible to use a covariance line on scale $h_{v}$ instead of the potential line connecting $Q^{\prime}$ to $Q$, so that we obtain again the bound improvement (A3.3). Therefore we obtain

$$
\begin{aligned}
& \int \prod_{j=2}^{s v} d \bar{z}_{j} d t_{j}|A(\ldots)| \\
& \quad<s_{v} ! \gamma^{\frac{h_{v}}{2}}\left(\sum_{j}\left|P_{v_{j}}\right|-\left|P_{v}\right|\right) C_{0}^{\left(\sum_{j}\left|P_{v_{j}}\right|-\left|P_{v}\right|\right)} C^{s_{v}-1} \gamma^{-2 h_{v}\left(s_{v}-1\right)} \gamma^{-h_{v}\left(1-\varepsilon_{0}\right)},
\end{aligned}
$$

where $\varepsilon_{0}$ is defined in (3.2) and takes in account the fact that the line which we integrate over can be soft or hard; if it is soft, the integration gives a factor $C h_{v}$. 2) Case $\left|P_{v_{1}}\right|=2$ : in this case, the situation is the following one:

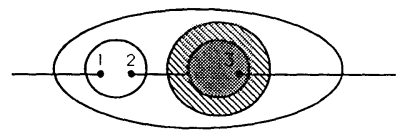


where the points 1 and 2 cannot be coincident, for, otherwise, the graph would be vanishing for parity reasons. We have labeled by 3 the point which the second external leg of $P_{v}$ comes out of. Then the following decomposition is always possible:

$$
\begin{aligned}
\mathscr{E}_{\left(h_{v}\right)}^{T}( & \left.: \tilde{\psi}^{\left(h_{v}\right)}\left(P_{v_{1}} \backslash Q_{v}^{(1)}\right):, \ldots,: \tilde{\psi}^{\left(h_{v}\right)}\left(P_{v_{s_{v}}} \backslash Q_{v}^{\left(s_{v}\right)}\right):\right) \\
= & \sum_{z_{1} \in \bigcup_{j=2}^{s_{v}} P_{v_{j}} \backslash Q_{v}^{(j)} \backslash 3} g^{\left(h_{v}\right)}\left(2-z_{1}\right) \\
& \times \mathscr{E}_{\left(h_{v}\right)}^{T}\left(: \tilde{\psi}^{\left(h_{v}\right)}\left(P_{v_{2}} \backslash Q_{v}^{(1)} \backslash\left[z_{1} \cap P_{v_{j}} \backslash Q_{v}^{(j)} \backslash 3\right]\right):, \ldots,\right. \\
& \left.\times: \tilde{\psi}^{\left(h_{v}\right)}\left(P_{v_{s_{v}}} \backslash Q_{v}^{\left(s_{v}\right)} \backslash\left[z_{1} \cap P_{v_{j}} \backslash Q_{v}^{(j)} \backslash 3\right]\right):\right)
\end{aligned}
$$

which doesn't allow an improvement of the bound, for the connection is realized only through hard lines in such a way that we have no soft line and exactly one hard line binding two subclusters. Nevertheless, in (A3.7) the term $\mathscr{E}_{\left(h_{v}\right)}(\ldots)$ can be studied like (A3.1). Again the estimate cannot be improved only if the subcluster which the external leg coming out of 2 goes into has two external legs. But in this case we can iterate the decomposition, until we reach a subcluster $P_{v_{m}}$, with $m \leq s$, which has more than two external legs. Then we have left a cluster (containing $s-m$ subclusters) which admits the decomposition (A3.3). We outline that no combinatorial problems arise, because the sum of terms we obtain after $m$ steps, is bounded by

$$
2(s-1) \ldots 2(s-m+1)
$$

(where the factor 2 can be eliminated) and the remaining truncated expectation yields a factorial $(s-m)$ ! so that we reobtain $C^{s} s$ !, like in the original bound. Therefore we can conclude that the bound cannot be improved only for a single contribution, given by

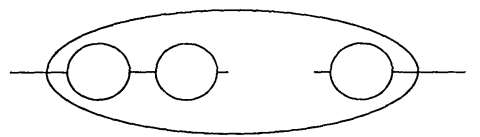

where $\left|P_{v_{2}}\right|=2 \forall i=1, \ldots, s_{v}$. Since the sets $P_{v_{i}}$ are fixed, either this term will be the only possible one (up to labeling of the sets $P_{v_{i}}$ and of the points which the external legs come out of) or there will be none.

We can now state the following result: $\forall P_{v}$ such that $\left|P_{v}\right|=2$, we have two possibilities:

i) $P_{v}$ is of the form (A3.8), and we don't decompose the truncated expectation (A3.1);

ii) $P_{v}$ has a different form (i.e. $\left|P_{v_{\imath}}\right|>2$ for any $i$ ) and we decompose

$$
\mathscr{E}_{\left(<h_{v}\right)}(\ldots) \mathscr{E}_{\left(h_{v}\right)}^{T}(\ldots)=E_{h_{v}}^{(1)}(\ldots)
$$

where $E^{(1)}(\ldots)$ will be a suitable function, which reduces to $A(\ldots)$ if $\left|P_{v_{\imath}}\right|>2$ and has a more involved but uniquely determined structure otherwise.

From the above discussion, it follows immediately that defining

$$
\begin{aligned}
\varrho_{v} & =\left(1-\varepsilon_{0}\right) \sum_{\bar{v} \geq v} \delta_{\left|P_{\bar{v}}\right|, 2}\left(1-\delta_{\left|P_{\bar{v}_{1}}\right|, 2} \ldots \delta_{\left|P_{\bar{v}_{s}}\right|, 2}\right) \\
& =\left(1-\varepsilon_{0}\right) \sum_{k=0}^{d_{1}(v)} \sum_{\substack{\bar{v} \geq v \\
d_{1}(\bar{v})=k}} \delta_{\left|P_{\bar{v}}\right|, 2}\left(1-\delta_{\left|P_{\bar{v}_{1}}\right|, 2} \ldots \delta_{\left|P_{\bar{v}_{\bar{v}}}\right|, 2}\right)
\end{aligned}
$$


the following bound holds

$$
\begin{aligned}
V\left(n, \gamma, P_{v_{0}}\right) \leq & \mathscr{C}^{n} \bar{\lambda}^{n} \sum_{\left\{h_{v}\right\}} \prod_{v \geq v_{0}}\left\{\sum_{P_{v_{1}} \ldots P_{v_{s}}} \sum_{Q_{v}^{(1)} \ldots Q_{v}^{(s)}} \gamma^{-\frac{1}{2}\left(\alpha_{v}+2 \varrho_{v}\right)\left(h_{v}-h_{v^{\prime}}\right)}\right. \\
& \left.\times \gamma^{-\frac{k}{2}\left(\alpha_{v_{0}}+2 \varrho_{v_{0}}\right)} e^{-\frac{\bar{\xi}}{2} d_{(k)}\left(\Delta_{1}, \ldots, \Delta_{\left|P_{v_{0}}\right|}\right)}\right\}
\end{aligned}
$$

and the inequalities

$$
\begin{gathered}
\varrho_{v^{\prime}} \geq \varrho_{v}, \\
\varrho_{v} \geq 1-\varepsilon_{0} \quad \forall\left|P_{v}\right|=2
\end{gathered}
$$

are an easy check if one use the second line of (A3.10).

Consider now again (A3.1) and let us suppose $\left|P_{v}\right|=2,4$, and $\left|P_{v_{j}}\right|>4$ for some $v_{j}, j=1, \ldots, s_{v}$, such that $P_{v_{j}} \cap P_{v} \neq \emptyset$, where $\left\{v_{j}\right\}_{j=1}^{s_{v}}$ is the collection of vertices immediately following $v$ moving forward along the tree.

If we choose a point which an external leg of $P_{v}$ comes out of, and we call 1 such a point and $P_{v_{1}}$ the subcluster which it belongs to, we can take obviously $\left|P_{v_{1}}\right|>4$. Since $\left|P_{v}\right|=4\left[\left|P_{v}\right|=2\right.$ ], at least two [four] lines coming out of $P_{v_{1}}$ are connected to the other subclusters $P_{v_{\imath}}, i \neq 1$, and at least one of these lines must be hard in order to guarantee the hard connectedness of the graph. For definiteness let us consider the case $\left|P_{v}\right|=4$ (but the same results hold also for the other cases).

We can proceed in the same way we have done above, and we find that (A3.1) admits the following graphic decomposition:
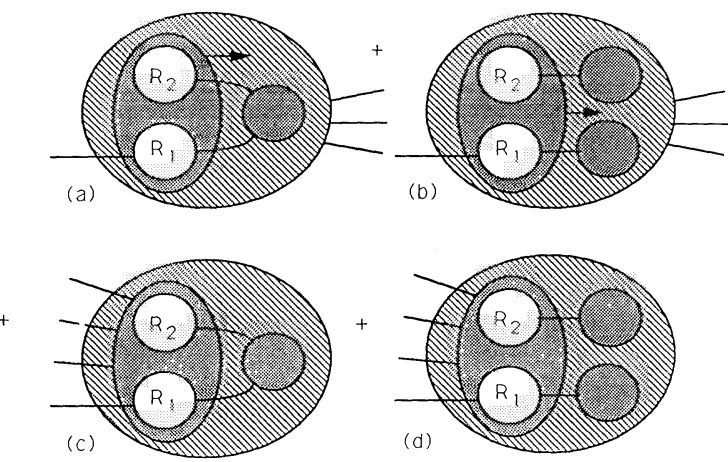

where in (a) and (b) we can suppose $\left|P_{v} \cap P_{v_{1}}\right|=1$, and the arrowed line represents a third line which we know for sure comes out of $P_{v_{1}}$ (i.e. of $R_{1}$ or $R_{2}$, according to the cases discussed above). We have therefore three lines, of which at least one is hard, and the others are such that we can use a space integration for one of them instead of a potential line (to which there corresponds a bounded and regular function). As far as the cases (c) and (d) are concerned, it can happen that the two external lines $l_{1}$ and $l_{2}$ coming out of, respectively, $R_{1}$ and $R_{2}$ are connected through a potential line which, therefore, becomes essential to the hard connectedness of the graph. Nevertheless, if at least one of the other subclusters $P_{v_{j}}, j \neq 1$, has $\left|P_{v_{j}}\right|=2$, then (A3.12) guarantees a gain on the scale difference $h_{v}-h_{v^{\prime}}$. Otherwise, if $\left|P_{v_{j}}\right|>2 \forall j \neq 1$, we can loose that gain, but in this case the topological structure of the graph is such that we can 
always delete a potential line emerging from a point

$$
Q^{*} \in \bigcup_{j=1}^{s_{v}} P_{v_{j}}
$$

which one of the two lines $l_{1}$ or $l_{2}$ (say $l_{1}$ ) is connected to, and perform the corresponding integration over the covariance line $g_{l_{1}}$. Then we can conclude that, in each case of the decomposition (A3.14), we can write an expression of the type (A3.9), where, obviously, now we have a different function $E^{(2)}$, so that

$$
\mathscr{E}_{\left(<h_{v}\right)}(\ldots) \mathscr{E}_{\left(h_{v}\right)}^{T}(\ldots)=E_{h_{v}}^{(2)}(\ldots),
$$

where $E^{(2)}$ can be thought of as a sum of terms which all admit an improvement of the superficial bound. In fact the only contribution that could be difficult to treat is that with $P_{v_{1}}$ connected only through a loop $g^{2}$ to the other subclusters, but it is absent for we cannot have a connection between $P_{v_{1}}$ and the other subclusters realized only by two lines.

In the end we obtain an improvement of the bound given by the factor

$$
\gamma^{-h_{v}\left(1-\varepsilon_{0}\right)}
$$

where $\varepsilon_{0}$ is due to the fact that the extra line which integrate over can be soft.

We outline again that the same results can be derived in the case $\left|P_{v}\right|=2$ too, so that, if we define

$$
\begin{aligned}
\sigma_{v}= & \sum_{\bar{v} \geq v}\left(\delta_{\left|P_{\bar{v}}\right|, 4}+\delta_{\left|P_{\bar{v}}\right|, 2}\right)\left[1-\prod_{\substack{j=1 \\
P_{\bar{v}_{j}} \cap P_{\bar{v}} \neq \emptyset}}^{s \bar{v}}\left(\delta_{\left|P_{\bar{v}_{j}}\right|, 4}+\delta_{\left|P_{\bar{v}_{j}}\right|, 2}\right)\right] \\
= & \sum_{k=0}^{d_{1}(v)} \sum_{\substack{\bar{v} \geq v \\
d_{1}(\bar{v})=k}}\left(\delta_{\left|P_{\bar{v}}\right|, 4}+\delta_{\left|P_{\bar{v}}\right|, 2}\right)\left[1-\prod_{\substack{j=1 \\
P_{\bar{v}_{j}} \cap P_{\bar{v}} \neq \emptyset}}^{s_{\bar{v}}}\left(\delta_{\left|P_{\bar{v}_{j}}\right|, 4}+\delta_{\left|P_{\bar{v}_{j}}\right|, 2}\right)\right] \\
= & \sum_{k=1}^{d_{1}(v)} \sum_{\substack{\bar{v} \geq v \\
d_{1}(\bar{v})=k}}\left(\delta_{\left|P_{\bar{v}}\right|, 4}+\delta_{\left|P_{\bar{v}}\right|, 2}\right)\left[1-\prod_{\substack{j=1 \\
P_{\bar{v}_{j}} \cap P_{\bar{v}} \neq \emptyset}}^{s_{\bar{v}}}\left(\delta_{\left|P_{\bar{v}_{j}}\right|, 4}+\delta_{\left|P_{\bar{v}_{j}}\right|, 2}\right)\right],
\end{aligned}
$$

and we apply successively the decompositions (A3.9) and (A3.15), obtaining the decomposition $E_{h_{v}}$, Lemma 4.1 holds.

\section{Appendix A4}

It is a standard result (see [GK, LL]) that

$$
\begin{aligned}
& \left|\mathscr{E}_{(h)}\left(\prod_{\jmath} \tilde{\psi}^{(h)}\left(P_{\jmath}\right)\right)\right| \leq \gamma^{\frac{h}{2} \sum_{\jmath}\left|P_{\jmath}\right|} C_{0}^{\sum_{\jmath}\left|P_{\jmath}\right|}, \\
& \left|\mathscr{E}_{(h)}^{T}\left(\tilde{\psi}^{(h)}\left(P_{1}\right), \ldots, \tilde{\psi}^{(h)}\left(P_{s}\right)\right)\right| \leq \gamma^{\frac{h}{2} \sum_{\jmath}\left|P_{\jmath}\right|} C_{T}^{\sum_{j}\left|P_{\jmath}\right|} \sum_{\mu} e^{-\xi d_{\mu(h)}\left(P_{1}, \ldots, P_{s}\right)}
\end{aligned}
$$


where $C_{0}, C_{T}$ are suitable constants. We want to generalize the above results to the case in which the fields appear with the Wick ordering, as in (2.9), (2.11), in order to obtain those inequalities and relate the new constants $C_{1}, C_{3}$ to the standard ones $C_{0}, C_{T}$.

We have

$$
\begin{aligned}
\mathscr{E}_{(h)}^{T}( & \left.: \tilde{\psi}^{(h)}\left(P_{1}\right):, \ldots,: \tilde{\psi}^{(h)}\left(P_{s}\right):\right) \\
= & \sum_{Y_{1} \subset P_{1}} \ldots \sum_{Y_{s} \subset P_{s}}(-1)^{\pi} \prod_{j=1}^{s} \mathscr{E}_{(h)}\left(\tilde{\psi}^{(h)}\left(P_{j} \backslash Y_{j}\right)\right) \\
& \times \mathscr{E}_{(h)}^{T}\left(\tilde{\psi}^{(h)}\left(P_{1}\right), \ldots, \tilde{\psi}^{(h)}\left(P_{s}\right)\right)
\end{aligned}
$$

and

$$
\begin{aligned}
\mathscr{E}_{(h)}\left(\prod_{j=1}^{s}: \tilde{\psi}^{(h)}\left(P_{j}\right):\right) \\
=\sum_{Y_{1} \subset P_{1}} \ldots \sum_{Y_{s} \subset P_{s}}(-1)^{\pi} \prod_{j=1}^{s} \mathscr{E}_{(h)}\left(\tilde{\psi}^{(h)}\left(P_{\jmath} \backslash Y_{\jmath}\right)\right) \mathscr{E}_{(h)}\left(\tilde{\psi}^{(h)}\left(Y_{j}\right)\right),
\end{aligned}
$$

where $\pi$ is a partiy which will turn to be inessential in the derivation of the following bounds.

Therefore (A4.1)-(A4.3) give

$$
\begin{aligned}
& \left|\mathscr{E}_{(h)}^{T}\left(: \tilde{\psi}^{(h)}\left(P_{1}\right):, \ldots,: \tilde{\psi}^{(h)}\left(P_{s}\right):\right)\right| \\
& \leq \sum_{q=1}^{\frac{1}{2}} \sum_{Y_{1} \subset P_{1} \ldots Y_{S} \subset P_{s}} \prod_{\jmath=1}^{s}\left|\mathscr{E}_{(h)}\left(\tilde{\psi}^{(h)}\left(P_{\jmath} \backslash Y_{\jmath}\right)\right)\right|\left|\mathscr{E}_{(h)}^{T}\left(\tilde{\psi}^{(h)}\left(P_{1}\right), \ldots, \tilde{\psi}^{(h)}\left(P_{s}\right)\right)\right| \\
& \sum_{j=1}^{s}\left|Y_{j}\right|=2 q \\
& \leq \sum_{q=1}^{\frac{1}{2} \sum_{j=1}^{s}\left|P_{j}\right|}\left(\begin{array}{c}
\frac{1}{2} \sum_{j=1}^{s}\left|P_{j}\right| \\
q
\end{array}\right) \gamma^{\frac{h}{2} \sum_{\jmath}\left(\left|P_{\jmath} \backslash Y_{\jmath}\right|+\left|Y_{\jmath}\right|\right)} \\
& \times C_{0}^{\sum_{j}^{j}\left|P_{\jmath} \backslash Y_{j}\right|} C_{T}^{\sum_{j}\left|Y_{\jmath}\right|} \sum_{\mu} e^{-\xi d_{\mu(h)}\left(P_{1}, \ldots, P_{s}\right)}
\end{aligned}
$$

which yields (2.11), with $C_{3}=2 \max \left\{C_{0}, C_{T}\right\}$.

By analogous reasoning it is straightforward to show that (A4.1), (A4.4) yield (2.9), with $C_{1}=2 C_{0}$. If we note that the proof of (2.10) is the same as the proof of (2.9), we can deduce from the above discussion that (2.10) holds too. Q.E.D.

Acknowledgements. This problem was originally proposed by Giovanni Gallavotti and Giosi Benfatto and we want to thank them for many useful discussions and suggestions. 


\section{References}

[BG] Benfatto, G., Gallavotti, G.: Perturbation theory of the Fermi surface in a quantum liquid. A general quasi particle formalism and one-dimensional systems. J. Stat. Phys. 59, 541-664 (1990)

[BGM] Benfatto, G., Gallavotti, G., Mastropietro, V.: Renormalization group and the Fermi surface in the Luttinger model. Phys. Rev. B 45, 5468 (1992)

[BGPS] Benfatto, G., Gallavotti, G., Procacci, A., Scoppola, B.: Beta function in one dimensional Fermi systems. Preprint

[CNPS] Cassandro, M., Nicoló, F., Perfetti, P., Scoppola, B.: The Gross-Neveu and Yukawa model. CARR reports $26(1990)$

[DM] Di Castro, C., Metzner, W.: Ward identities and beta function in the Luttinger liquid. Phys. Rev. Lett. 67, 3852-3855 (1991)

[G1] Gallavotti, G.: Renormalization theory and ultraviolet stability for scalar fields via renormalization group methods. Rev. Mod. Phys. 57, 471-562 (1985)

[G2] Gallavotti, G.: Renormalization group. Troisieme Cycle de la Physique, Lausanne (1990)

[GK] Gawedzki, K., Kupiainen, A.: Gross-Neveu model through convergent perturbative expansions. Commun. Math. Phys. 102, 1-30 (1985)

[GN] Gallavotti, G., Nicoló, F.: Renormalization theory in four-dimensional scalar fields. I. Commun. Math. Phys. 100, 545-590 (1985); II. Commun. Math. Phys. 101, 247-282 (1985)

[L] Luttinger, J.M.: An exactly soluble model of a many-fermion system. J. Math. Phys. 4, 1154-1162 (1963)

[LL] Lesniewski, A.: Effective action on the Yukawa 2 quantum field theory. Commun. Math. Phys. 108, 437-467 (1987)

[ML] Mattis, D.G., Lieb, E.H.: Exact solution of a many-fermion system and its associated boson field. J. Math. Phys. 6, 304-312 (1965)

Communicated by G. Felder 
